\title{
The Impact of Cross-Species Gene Flow on Species Tree Estimation
}

\author{
XiYun JiaO ${ }^{1}$, Thomas Flouris ${ }^{1}$, Bruce Rannala ${ }^{2}$ And Ziheng YAng ${ }^{1, *}$ \\ ${ }^{1}$ Department of Genetics, University College London, Gower Street, London WCIE 6BT, UK \\ ${ }^{2}$ Department of Evolution and Ecology, University of California, Davis, CA 95616, USA \\ * Ziheng Yang, Department of Genetics, University College London, Gower Street, London WCIE 6BT, UK. \\ Phone: +44 20 7679-4379 (z.yang@ucl.ac.uk)
}

\begin{abstract}
Recent analyses of genomic sequence data suggest cross-species gene flow is common in both plants and animals, posing challenges to species tree estimation. We examine the levels of gene flow needed to mislead species tree estimation with three species and either episodic introgressive hybridization or continuous migration between an outgroup and one ingroup species. Several species tree estimation methods are examined, including the majority-vote method based on the most common gene tree topology (with either the true or reconstructed gene trees used), the UPGMA method based on the average sequence distances (or average coalescent times) between species, and the full-likelihood method based on multi-locus sequence data. Our results suggest that the majority-vote method based on gene tree topologies is more robust to gene flow than the UPGMA method based on coalescent times and both are more robust than likelihood assuming a multispecies coalescent (MSC) model with no cross-species gene flow. Comparison of the continuous migration model with the episodic introgression model suggests that a small amount of gene flow per generation can cause drastic changes to the genetic history of the species and mislead species tree methods, especially if the species diverged through radiative speciation events. Estimates of parameters under the MSC with gene flow suggest that African mosquito species in the Anopheles gambia species complex constitute such an example of extreme impact of gene flow on species phylogeny.
\end{abstract}

Key words: Introgression, migration, species tree, multispecies coalescent, IM, MSci

Cross-species hybridization or introgression has long been recognized as an important process that generates biological diversity in plants (e.g., Anderson, 1949; Mallet, 2007). Analyses of genomic data in the past few years suggest that introgression is also common in animals (Mao et al., 2018; Ellegren et al., 2012; Kumar et al., 2017; Wu et al., 2018; Chan et al., 2013), including humans and their close relatives (Nielsen et al., 2017). Introgression may involve either sister or non-sister species (e.g., Mallet et al., 2016) and may play an important role in the speciation process (Mallet et al., 2016; Martin and Jiggins, 2017). Introgression, together with deep coalescence (also known as incomplete lineage sorting, ILS), may cause difficulties for species tree reconstruction (Maddison, 1997; Nichols, 2001). In extreme cases, the whole genome, and in particular the autosomes, are affected by such pervasive gene flow that they do not reflect the species phylogeny anymore. This appears, for example, to be the case with the Anopheles gambiae species complex, in which the autosomes suggest different species 
relationships from the $\mathrm{X}$ chromosome, which, being apparently enriched with sterility genes and resistant to cross-species gene flow, reflects the true history of species divergences (Fontaine et al., 2015). The Heliconius butterflies appear to be another such case, with the Z chromosome favoring different phylogenies from the autosomes (Edelman et al., 2019). In both examples, the species arose through a rapid succession of speciation events, generating species phylogenies with very short interior branches, which are challenging to reconstruct even without cross-species gene flow.

A number of methods have been developed to detect gene flow across species using genetic sequence data, including population genetic methods based on $F_{\text {st }}$ and summary methods that make use of observed site patterns (Green et al., 2010; Durand et al., 2011) or estimated gene tree topologies (Yu et al., 2012, 2014; Yu and Nakhleh, 2015; Solis-Lemus and Ane, 2016; Wen et al., 2016). Full likelihood methods based on sequence alignments (Hey and Nielsen, 2004; Hey et al., 2018; Wen and Nakhleh, 2018; Zhang et al., 2018) are also being actively developed. See Degnan (2018) and Folk et al. (2018) for recent reviews.

Here we consider the question of how much gene flow is sufficient to mislead species tree estimation methods that accommodate the coalescent process but not gene flow. As discussed by Folk et al. (2018), the impact of gene flow on the tree of life is an important topic worth serious study. We focus on the case of three species with sequences evolving under the molecular clock and are specifically interested in closely related species, for which gene flow may be a major concern. We consider two distinct models of gene flow, both of which accommodate the multispecies coalescent (MSC). The model of isolation with migration (IM) assumes continuous migration, with the species exchanging migrants at a certain rate in every generation (see Fig. 1b\&d for examples) (Hey and Nielsen, 2004; Hey, 2010). The model of multispecies coalescent with introgression (MSci) assumes episodic introgression or hybridization, with introgressions occurring at fixed time points in the past (e.g., Fig. 1a\&c) (Yu et al., 2012, 2014; Wen and Nakhleh, 2018). The MSci model was called the "multispecies network coalescent" (MSNC) by Wen and Nakhleh (2018). As the term "network" has been used to refer to both biological and nonbiological processes (Solis-Lemus and Ane, 2016; Degnan, 2018), we follow Degnan (2018) and use the more-expressive term "MSC with introgression" to highlight the two major factors in the model: coalescent and introgression. We consider two scenarios of gene flow on the species tree $(A, B, C))$. Inflow is introgression (or migration) from the outgroup species $A$ to the ingroup species $C$, and outflow is in the reverse direction. In both scenarios, gene flow makes species $A$ and $C$ look similar, potentially misleading species tree methods to infer the incorrect tree $(B,(C, A))$.

Previously Leaché et al. (2014) used computer simulation to examine the impact of continuous migration on species tree inference, finding that the effects depend on the species tree topology and the mode of gene flow. For example, while gene flow between non-sister species might mislead species tree inference, gene flow between sister species actually made it easier to infer the species tree. Solis-Lemus et al. (2016) used simulation to study the statistical consistency of several species tree methods that ignore gene flow, including ASTRAL (Mirarab et al., 2014) and NJST (Liu and Yu, 2011), when the model assumes cross-species introgression. A method was considered inconsistent if the probability of retrieving the correct species tree fails to increase when the number of gene trees (the number of loci) increases. The approach we take here is largely analytical, not affected by sampling errors in simulation. Hahn and Nakhleh (2016, fig. 3) calculated gene-tree probabilities under an introgression model, arguing that the concept of a species tree is poorly defined when there is gene flow. Long and Kubatko (2018) studied the probabilities of gene tree topologies under a model of isolation with initial migration (IIM) (Wilkinson-Herbots, 2012) for three species, in which there is initial gene flow between sister species after their divergence. One might expect gene flow between sister species to make them appear more similar, making it easier to infer the species tree, but surprisingly with different population sizes, gene flow between sister species can cause the most probable gene tree topology to differ from the species tree, leading to so-called "anomalous gene trees". 
Here we study the asymptotic behavior of several species tree inference methods when there is gene flow affecting non-sister species. All the methods ignore gene flow but are statistically consistent in the case of three species when there is deep coalescence but no gene flow. We consider both continuous migration and episodic introgression. The first method is the majority-vote method of using the most common (true) gene tree topology as the estimate of the species tree. This is known to be statistically consistent in the case of three species and three sequences per locus when there is no gene flow, with the estimated species tree approaching the true tree when the number of loci approaches infinity (Hudson, 1983). We derive the distribution of the true gene tree topologies under each model of gene flow, and examine the impact of phylogenetic reconstruction errors when the estimated gene trees are used to estimate the species tree. Use of estimated gene trees is known to be consistent in the case of three species when the model involves coalescent but no gene flow (Yang, 2002). Next we consider the UPGMA method, which uses the average sequence distance between species (or the average coalescent time between species since we assume the molecular clock) to infer the species tree (Liu et al., 2009). This is equivalent to calculating sequence distances using concatenated data followed by UPGMA reconstruction of the species tree. This method is known to be consistent in the case of three species (Liu et al., 2009). Finally, we consider the ML method of species tree estimation, which averages over the gene trees and branch lengths and thus accounts for phylogenetic reconstruction errors (Xu and Yang, 2016; Yang, 2002). While full-likelihood methods of species tree estimation under the MSC applied to multilocus sequence alignments, including maximum likelihood (ML) (Yang, 2002; Zhu and Yang, 2012) and Bayesian inference (Liu and Pearl, 2007; Heled and Drummond, 2010; Yang and Rannala, 2014), are analytically intractable, the 3S program has an efficient ML implementation that can handle thousands of loci (Yang, 2002; Dalquen et al., 2017). We thus use 3 s to analyze tens of thousands of loci to approximate the case of infinite data. Note that our interest is in the consistency or inconsistency of each species tree estimation method in the face of gene flow as the number of loci approaches infinity. To our knowledge, this represents the first analysis of full-likelihood methods based on sequence alignments, which may be expected to make the most efficient use of information in the sequence data and to have statistically optimal performance when the model is correct. The performance of likelihood methods when the model is mis-specified is unknown. As pointed out by Solis-Lemus et al. (2016), statistical consistency is conventionally defined under the true model, but here we follow the tradition in statistical phylogenetics to examine the impact of model violations.

We note that with cross-species gene flow, the concept of the true species tree may be ambiguous (Hahn and Nakhleh, 2016). One strategy, adopted in PhyloNetworks (Solis-Lemus et al., 2017), is to use the introgression probability to define the major species tree, which is the species tree represented by parental branches at the hybridization nodes with contribution probabilities $>\frac{1}{2}$. For example, in Fig. 1a, the true species tree is $(A(C B))$ if $\varphi<\frac{1}{2}$ and $((A C) B)$ if $\varphi>\frac{1}{2}$. With this definition, the true species tree changes when the introgression probability increases from below 0.5 to above 0.5 , with the truth always on the winning side. Another strategy is to start with a species tree and add gene flow onto it, with the true species tree to be always the starting species tree. In this paper, we use the second strategy, motivated by the inferred pattern of gene flow in the Anopheles gambiae species group (see Discussion).

\section{THEORY}

We consider two gene flow scenarios: "inflow" where there is gene flow (introgression or migration) from the outgroup species $A$ to the ingroup species $C$ on the species tree $(A,(B, C))$ (Fig. 1a \& b) and "outflow" in the opposite direction (Fig. 1c \& d). Both the species divergence times $\left(\tau_{R}, \tau_{S}\right.$, and $\left.\tau_{T}\right)$ and the population size parameters $(\theta \mathrm{s})$ in the model are measured by the expected number of mutations/substitutions per site. The data consist of multiple loci, with three sequences - one from each of the three species at each locus $(a, b, c)$. The possible gene trees at 
each locus are $G_{1}=(a,(b, c))$, which matches the species tree; $G_{2}=(b,(c, a))$, which reflects the introgression or migration pattern; and $G_{3}=(c,(a, b))$ which matches neither. Sequence data are then analyzed to infer the species tree under the multispecies coalescent (MSC) model (assuming no gene flow). We derive analytical results for two simple methods of species tree estimation assuming no gene flow: (1) the majority-vote method for which the correct species tree is inferred if $\mathbb{P}\left(G_{1}\right)>\mathbb{P}\left(G_{2}\right)$ and (2) the UPGMA method using the average coalescent times across loci (Liu et al., 2009) for which the correct species tree is inferred if $\mathbb{E}\left(t_{b c}\right)<\mathbb{E}\left(t_{a c}\right)$. Initially, we ignore sampling errors in the gene trees or the estimated sequence distances. We study the asymptotic behavior of the two methods as the number of loci approaches infinity. We derive results for the two methods under both the MSci and IM models.

We note that the analytical results derived here apply to the specific scenarios of species tree and gene flow. Even for the case of three species, we do not consider gene flow between sister species (Dalquen et al., 2017; Long and Kubatko, 2018) or bidirectional gene flow $(A \leftrightarrow C)$. Larger species trees with more than three species and more complex gene flow scenarios involving more than one pair of species will add much complexity to the analysis (Zhu et al., 2016; Zhu and Degnan, 2017).

\section{Gene flow under the MSci model}

Here, we consider properties of the majority-vote and UPGMA methods for data arising under a MSci model of admixture. Referring to Figure 1 we define

$$
P_{S}=\mathrm{e}^{-\frac{2}{\theta_{S}}\left(\tau_{R}-\tau_{S}\right)} \text { and } P_{T}=\mathrm{e}^{-\frac{2}{\theta_{T}}\left(\tau_{R}-\tau_{T}\right)}
$$

to be the probability that two sequences entering either species $S$, or species $T$, do not coalesce in that species and instead both enter the ancestral species (species $R$ for both). Note that $\frac{2}{\theta_{S}}\left(\tau_{R}-\tau_{S}\right)$ and $\frac{2}{\theta_{T}}\left(\tau_{R}-\tau_{T}\right)$ are known as the branch lengths in coalescent units in Figure 1 .

We first consider properties of the methods with data generated by inflow.

UPGMA Method with Inflow Here we derive results for a model assuming instantaneous introgression from $A$ to $C$ (inflow), with the introgression probability $\varphi$. Sequences $a$ and $b$ can coalesce in $R$ only, and the coalescent time has the exponential density $f\left(t_{a b}\right)=\frac{2}{\theta_{R}} \mathrm{e}^{-\frac{2}{\theta_{R}}\left(t_{a b}-\tau_{R}\right)}$ with expectation $\mathbb{E}\left(t_{a b}\right)=\tau_{R}+\frac{\theta_{R}}{2}$. Sequences $a$ and $c$ can coalesce in population $S$ as well as $R$, while sequences $b$ and $c$ can coalesce in species $T$ as well as $R$. This means that $\mathbb{E}\left(t_{a b}\right)>\max \left\{\mathbb{E}\left(t_{a c}\right), \mathbb{E}\left(t_{b c}\right)\right\}$. The probability density of the coalescent time $t_{a c}$ is

$$
f\left(t_{a c}\right)= \begin{cases}\varphi \frac{2}{\theta_{S}} \mathrm{e}^{-\frac{2}{\theta_{S}}\left(t_{a c}-\tau_{S}\right)}, & \text { if } \tau_{S}<t_{a c}<\tau_{R}, \\ \varphi P_{S} \frac{2}{\theta_{R}} \mathrm{e}^{-\frac{2}{\theta_{R}}\left(t_{a c}-\tau_{R}\right)}+(1-\varphi) \frac{2}{\theta_{R}} \mathrm{e}^{-\frac{2}{\theta_{R}}\left(t_{a c}-\tau_{R}\right)}, & \text { if } t_{a c}>\tau_{R}\end{cases}
$$

with expectation

$$
\mathbb{E}\left(t_{a c}\right)=\varphi\left[\tau_{S}+\frac{\theta_{S}}{2}+P_{S}\left(\frac{\theta_{R}}{2}-\frac{\theta_{S}}{2}\right)\right]+(1-\varphi)\left(\tau_{R}+\frac{\theta_{R}}{2}\right) .
$$

Similarly, sequences $b$ and $c$ can coalesce in species $T$ and $R$, so we have the density

$$
f\left(t_{b c}\right)= \begin{cases}(1-\varphi) \frac{2}{\theta_{T}} \mathrm{e}^{-\frac{2}{\theta_{T}}\left(t_{b c}-\tau_{T}\right)}, & \text { if } \tau_{T}<t_{b c}<\tau_{R}, \\ (1-\varphi) P_{T} \frac{2}{\theta_{R}} \mathrm{e}^{-\frac{2}{\theta_{R}}\left(t_{b c}-\tau_{R}\right)}+\varphi \frac{2}{\theta_{R}} \mathrm{e}^{-\frac{2}{\theta_{R}}\left(t_{b c}-\tau_{R}\right)}, & \text { if } t_{b c}>\tau_{R},\end{cases}
$$




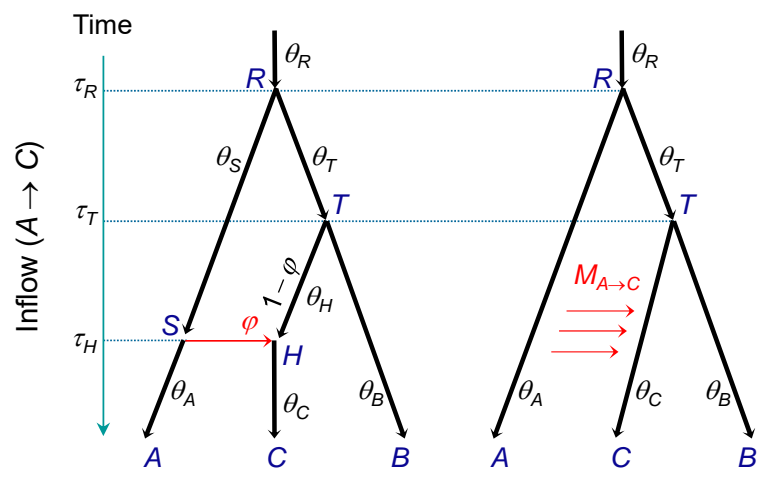

(a)

(b)

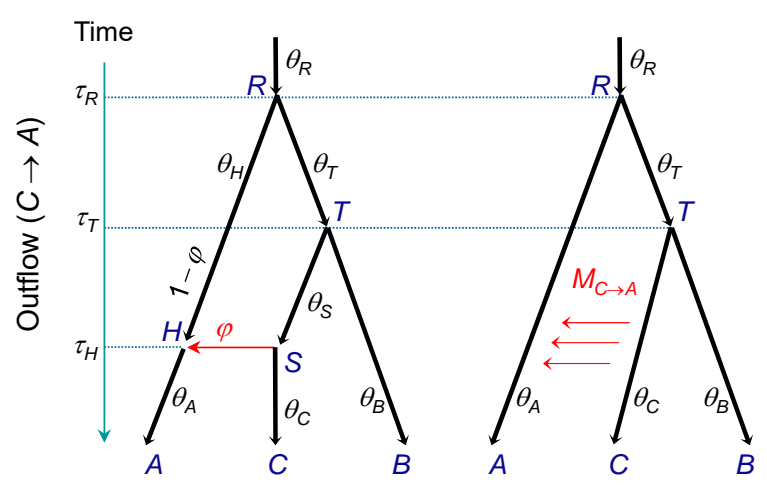

(c)

(d)

Fig. 1. Species tree $(A,(B, C))$, with introgression (a and $\mathbf{c}$ ) or migration (b and $\mathbf{d}$ ) from the outgroup species $A$ to the ingroup species $C$ (inflow, $\mathbf{a}$ and $\mathbf{b}$ ) or in the reverse direction (outflow, $\mathbf{c}$ and $\mathbf{d}$ ). In the MSci models, an introgression or hybridization event occurs at time $\tau_{H}=\tau_{S}$, and the hybrid species $H$ consists of a proportion $\varphi$ of introgressed individuals. In the IM model, gene flow occurs continuously (i.e., in every generation) over the time period $\left(0, \tau_{T}\right)$. The migration rate in $\mathbf{b}$ is defined as $M_{A \rightarrow C}=N_{C} m_{A \rightarrow C}$, where $N_{C}$ is (effective) population size of species $C$ and $m_{A \rightarrow C}$ is the proportion of immigrants (from species $A$ ) in the receiving population $C$, so that $M_{A \rightarrow C}$ is the expected number of migrants from $A$ to $C$ per generation. Migration rate in $\mathbf{d}$ is defined similarly as $M_{C \rightarrow A}=N_{A} m_{C \rightarrow A}$. In this paper, the backbone tree represented by black thick branches is considered the true species tree, irrespective of the strength of gene flow (or the values of $\varphi$ or $M$ ).

with expectation

$$
\mathbb{E}\left(t_{b c}\right)=(1-\varphi)\left[\tau_{T}+\frac{\theta_{T}}{2}+P_{T}\left(\frac{\theta_{R}}{2}-\frac{\theta_{T}}{2}\right)\right]+\varphi\left[\tau_{R}+\frac{\theta_{R}}{2}\right] .
$$

Then $\mathbb{E}\left(t_{b c}\right)>\mathbb{E}\left(t_{a c}\right)$ and the UPGMA method based on average coalescent times infers an incorrect species tree if and only if

$$
\varphi>\frac{1}{1+\frac{\tau_{R}-\tau_{S}+\frac{1}{2}\left(1-P_{S}\right)\left(\theta_{R}-\theta_{S}\right)}{\tau_{R}-\tau_{T}+\frac{1}{2}\left(1-P_{T}\right)\left(\theta_{R}-\theta_{T}\right)}} .
$$

Majority-Vote Method with Inflow If sequences $b$ and $c$ coalesce in population $T$, the gene tree will be $G_{1}=(a,(b, c))$, while if $a$ and $c$ coalesce in population $S$, the gene tree will be 
the $G_{2}=(b,(c, a))$. If neither of those events occurs, both coalescent events for the three sequences will occur in species $R$ and the three gene trees will occur with equal probabilities. Thus $\mathbb{P}\left(G_{3}\right)<\min \left\{\mathbb{P}\left(G_{1}\right), \mathbb{P}\left(G_{2}\right)\right\}$. We have

$$
\begin{aligned}
& \mathbb{P}\left(G_{1}\right)=\frac{1}{3} \varphi P_{S}+(1-\varphi)\left(1-P_{T}+\frac{1}{3} P_{T}\right), \\
& \mathbb{P}\left(G_{2}\right)=\varphi\left(1-P_{S}+\frac{1}{3} P_{S}\right)+\frac{1}{3}(1-\varphi) P_{T}, \\
& \mathbb{P}\left(G_{3}\right)=\frac{1}{3}\left[\varphi P_{S}+(1-\varphi) P_{T}\right]=1-\mathbb{P}\left(G_{1}\right)-\mathbb{P}\left(G_{2}\right) .
\end{aligned}
$$

For example, gene tree $G_{1}$ results from sequences $b$ and $c$ coalescing first. If sequence $c$ enters species $S$ (which happens with probability $\varphi$ ), this can occur only if sequences $c$ and $a$ do not coalesce in species $S$. This is the first term, $\varphi P_{S} \cdot \frac{1}{3}$, in $\mathbb{P}\left(G_{1}\right)$. If sequence $c$ enters species $H$ (which happens with probability $1-\varphi$ ), sequences $b$ and $c$ can coalesce in species $T$ or $R$. Hence the second term $(1-\varphi)\left(1-P_{T}+\frac{1}{3} P_{T}\right)$.

Thus $\mathbb{P}\left(G_{1}\right)<\mathbb{P}\left(G_{2}\right)$ and the majority-vote method based on the most common gene tree infers an incorrect species tree if and only if

$$
\varphi>\frac{1-P_{T}}{2-P_{S}-P_{T}}=\frac{1}{1+\frac{1-P_{S}}{1-P_{T}}} .
$$

This can also be obtained by noting that $\mathbb{P}\left(G_{1}\right)<\mathbb{P}\left(G_{2}\right)$ if and only if the probability that sequences $b$ and $c$ coalesce in population $T$ is smaller than the probability that sequences $a$ and $c$ coalesce in population $S$ : that is, if $(1-\varphi)\left(1-P_{T}\right)<\varphi\left(1-P_{S}\right)$.

Note that the gene tree probabilities (Equation 1.6) and the $\varphi$ limit based on them (Equation 1.7) depend on only the internal branch lengths (in coalescent units) on the species tree, but not the species divergence times and population sizes. The $\varphi$ limits for both gene tree probabilities and the average coalescent times (Equations 1.7 and 1.5) depend on the gene tree topologies and coalescent times but not the mutation rate: they are functions of $\tau / \theta$ ratios and not of $\tau \mathrm{s}$ and $\theta$ s individually.

UPGMA Method with Outflow Here we derive results for a model assuming instantaneous introgression from $C$ to $A$ (outflow) with introgression probability $\varphi$. Referring to Figure 1c we define

$$
P_{S}^{*}=\mathrm{e}^{-\frac{2}{\theta_{S}}\left(\tau_{T}-\tau_{S}\right)}
$$

to be the probability that two sequences entering population $S$ do not coalesce in that population and instead enter its ancestor (population $T$ ) (Fig. 1c). Sequences $a$ and $c$ can coalesce in populations $S, T$ and $R$, and sequences $b$ and $c$ or $a$ and $b$ can coalesce in species $T$ and $R$. The probability density of the coalescent time $t_{a c}$ is

$$
f\left(t_{a c}\right)= \begin{cases}\varphi \frac{2}{\theta_{S}} \mathrm{e}^{-\frac{2}{\theta_{S}}\left(t_{a c}-\tau_{S}\right)}, & \text { if } \tau_{S}<t_{a c}<\tau_{T}, \\ \varphi P_{S}^{*} \frac{2}{\theta_{T}} \mathrm{e}^{-\frac{2}{\theta_{T}}\left(t_{a c}-\tau_{T}\right)}, & \text { if } \tau_{T}<t_{a c}<\tau_{R}, \\ {\left[\varphi P_{S}^{*} P_{T}+(1-\varphi)\right] \frac{2}{\theta_{R}} \mathrm{e}^{-\frac{2}{\theta_{R}}\left(t_{a c}-\tau_{R}\right)},} & \text { if } t_{a c}>\tau_{R}\end{cases}
$$

with expectation

$$
\mathbb{E}\left(t_{a c}\right)=\varphi\left[\tau_{S}+\frac{\theta_{S}}{2}+P_{S}^{\star}\left(\frac{\theta_{T}}{2}-\frac{\theta_{S}}{2}\right)+P_{S}^{\star} P_{T}\left(\frac{\theta_{R}}{2}-\frac{\theta_{T}}{2}\right)\right]+(1-\varphi)\left[\tau_{R}+\frac{\theta_{R}}{2}\right] .
$$


The coalescent time between sequences $b$ and $c$ has the density

$$
f\left(t_{b c}\right)= \begin{cases}\frac{2}{\theta_{T}} \mathrm{e}^{-\frac{2}{\theta_{T}}\left(t_{b c}-\tau_{T}\right)}, & \text { if } \tau_{T}<t_{b c}<\tau_{R}, \\ P_{T} \frac{2}{\theta_{R}} \mathrm{e}^{-\frac{2}{\theta_{R}}\left(t_{b c}-\tau_{R}\right)}, & \text { if } t_{b c}>\tau_{R}\end{cases}
$$

with expectation

$$
\mathbb{E}\left(t_{b c}\right)=\tau_{T}+\frac{\theta_{T}}{2}+P_{T}\left(\frac{\theta_{R}}{2}-\frac{\theta_{T}}{2}\right)
$$

The probability density of the coalescent time $t_{a b}$ is

$$
f\left(t_{a b}\right)= \begin{cases}\varphi \frac{2}{\theta_{T}} \mathrm{e}^{-\frac{2}{\theta_{T}}\left(t_{a b}-\tau_{T}\right)}, & \text { if } \tau_{T}<t_{a b}<\tau_{R} \\ {\left[\varphi P_{T}+(1-\varphi)\right] \frac{2}{\theta_{R}} \mathrm{e}^{-\frac{2}{\theta_{R}}\left(t_{a b}-\tau_{R}\right)},} & \text { if } t_{a b}>\tau_{R}\end{cases}
$$

with the expectation

$$
\mathbb{E}\left(t_{a b}\right)=\varphi\left[\tau_{T}+\frac{\theta_{T}}{2}+P_{T}\left(\frac{\theta_{R}}{2}-\frac{\theta_{T}}{2}\right)\right]+(1-\varphi)\left(\tau_{R}+\frac{\theta_{R}}{2}\right) .
$$

It is easy to see that $\mathbb{E}\left(t_{a b}\right)>\max \left\{\mathbb{E}\left(t_{a c}\right), \mathbb{E}\left(t_{b c}\right)\right\}$. Then $\mathbb{E}\left(t_{b c}\right)>\mathbb{E}\left(t_{a c}\right)$ and the UPGMA method infers an incorrect species tree if and only if

$$
\varphi>\frac{\tau_{R}-\tau_{T}+\frac{1}{2}\left(1-P_{T}\right)\left(\theta_{R}-\theta_{T}\right)}{\tau_{R}-\tau_{S}+\frac{1}{2}\left(\theta_{R}-\theta_{S}\right)-\frac{1}{2} P_{S}^{\star}\left(\theta_{T}-\theta_{S}\right)-\frac{1}{2} P_{T} P_{S}^{\star}\left(\theta_{R}-\theta_{T}\right)} .
$$

Majority-Vote Method with Outflow When we trace the genealogy of sequences $a, b$, and $c$ backward in time, sequence $a$ may enter species $S$ (with probability $\varphi$ ) or species $H$ (with probability $1-\varphi$ ). Consider the first case, of sequence $a$ entering species $S$. If $a$ and $c$ coalesce in population $S$, the gene tree will be $G_{2}$. Otherwise, the two coalescent events for the three sequences can occur in either $T$ or $R$ and the three gene trees occur with equal probabilities. In the second case, sequence $a$ enters species $H$. Then if $b$ and $c$ coalesce in population $T$, the gene tree will be $G_{1}$, and otherwise, both coalescent events for the three sequences will occur in $R$ with equal probabilities for the three gene trees. This means that $G_{3}$ is the least frequent gene tree, with $\mathbb{P}\left(G_{3}\right)<\min \left\{\mathbb{P}\left(G_{1}\right), \mathbb{P}\left(G_{2}\right)\right\}$. We have

$$
\begin{aligned}
& \mathbb{P}\left(G_{1}\right)=\frac{1}{3} \varphi P_{S}^{\star}+(1-\varphi)\left(1-P_{T}+\frac{1}{3} P_{T}\right), \\
& \mathbb{P}\left(G_{2}\right)=\varphi\left(1-P_{S}^{\star}+\frac{1}{3} P_{S}^{\star}\right)+\frac{1}{3}(1-\varphi) P_{T}, \\
& \mathbb{P}\left(G_{3}\right)=\frac{1}{3}\left[\varphi P_{S}^{\star}+(1-\varphi) P_{T}\right]=1-\mathbb{P}\left(G_{1}\right)-\mathbb{P}\left(G_{2}\right) .
\end{aligned}
$$

Thus $\mathbb{P}\left(G_{1}\right)<\mathbb{P}\left(G_{2}\right)$, i.e., the majority-vote method infers an incorrect species tree, if and only if

$$
\varphi>\frac{1-P_{T}}{2-P_{S}^{\star}-P_{T}}=\frac{1}{1+\frac{1-P_{S}^{\star}}{1-P_{T}}} .
$$




\section{Gene Flow under the IM Model}

UPGMA Method with Inflow We first consider an isolation-with-migration (IM) model with inflow. Define the migration rate (in forward-time) from species $A$ to $C$ under the IM model to be $M_{A C}=m_{A C} N_{C}$ migrants per generation (Fig. 1b). When we trace the genealogy of the sample backwards in time, the process of coalescence and migration during time interval $\left(0, \tau_{T}\right)$ can be described by a Markov chain with three states: $S_{a b c}, S_{a a b}$ and $S_{a b}$ (Hobolth et al., 2011; Zhu and Yang, 2012). Here $S_{a b c}$ is the initial state with the three sequences in the three populations, $S_{a a b}$ is the state after sequence $c$ enters species $A$ (tracing the genealogy backwards in time), with two sequences in $A$ and a third in $B$, and $S_{a b}$ is the state after sequence $c$ enters $A$ and coalesces with sequence $a$, so that two sequences remain in the sample.

As the model assumes unidirectional migration, the only migration possible is from $C$ to $A$ (when time runs backwards), at the rate $m_{A C}$ per generation. The only coalescent possible during the time epoch $\left(0, \tau_{T}\right)$ is between $a$ and $c$ in population $A$, at the rate $1 /\left(2 N_{A}\right)$ per generation (Fig. 1b). Divide both rates by the mutation rate $\mu$ per generation so that one time unit is the expected time taken to accumulate one mutation per site. Then the migration rate becomes $w_{A C}=m_{A C} / \mu=4 M_{A C} / \theta_{C}$, and the coalescent rate in species $A$ becomes $2 / \theta_{A}$. Thus the backward process of migration and coalescence can be described by a Markov chain with the generator matrix $Q$

\begin{tabular}{c|ccc} 
& $S_{a b c}$ & $S_{a a b}$ & $S_{a b}$ \\
\hline$S_{a b c}$ & $-w_{A C}$ & $w_{A C}$ & 0 \\
$S_{a a b}$ & 0 & $-\frac{2}{\theta_{A}}$ & $\frac{2}{\theta_{A}}$ \\
$S_{a b}$ & 0 & 0 & 0
\end{tabular}

The eigenvalues of $Q$ are $\lambda_{1}=0, \lambda_{2}=-\frac{2}{\theta_{A}}$, and $\lambda_{3}=-w_{A C}$. The transition probability matrix, $P(t)=\exp (Q t)$, is

\begin{tabular}{c|ccc} 
& $S_{a b c}$ & $S_{a a b}$ & $S_{a b}$ \\
\hline$S_{a b c}$ & $\mathrm{e}^{-w_{A C} t}$ & $\frac{\theta_{A} w_{A C}}{2-\theta_{A} w_{A C}}\left(\mathrm{e}^{-w_{A C} t}-\mathrm{e}^{-\frac{2}{\theta_{A}} t}\right)$ & $1-\frac{2}{2-\theta_{A} w_{A C}} \mathrm{e}^{-w_{A C} t}+\frac{\theta_{A} w_{A C}}{2-\theta_{A} w_{A C}} \mathrm{e}^{-\frac{2}{\theta_{A}} t}$ \\
$S_{a a b}$ & 0 & $\mathrm{e}^{-\frac{2}{\theta_{A}} t}$ & $1-\mathrm{e}^{-\frac{2}{\theta_{A}} t}$ \\
$S_{a b}$ & 0 & 0 & 1
\end{tabular}

Sequences $a$ and $b$ can coalesce in population $R$ only, with the coalescent time having an exponential distribution $f\left(t_{a b}\right)=\frac{2}{\theta_{R}} \mathrm{e}^{-\frac{2}{\theta_{R}}\left(t_{a b}-\tau_{R}\right)}, t_{a b}>\tau_{R}$, with expectation $\mathbb{E}\left(t_{a b}\right)=\tau_{R}+\frac{\theta_{R}}{2}$. Sequences $a$ and $c$ can coalesce in both $A$ and $R$, while sequences $b$ and $c$ can coalesce in both $T$ and $R$. Thus $\mathbb{E}\left(t_{a b}\right)>\max \left\{\mathbb{E}\left(t_{a c}\right), \mathbb{E}\left(t_{b c}\right)\right\}$.

The probability density of coalescent time $t_{a c}$ is

$$
f\left(t_{a c}\right)= \begin{cases}P_{a b c, a a b}\left(t_{a c}\right) \frac{2}{\theta_{A}}, & \text { if } 0<t_{a c}<\tau_{T} \\ P_{a b c, a a b}\left(\tau_{T}\right) \frac{2}{\theta_{A}} \mathrm{e}^{-\frac{2}{\theta_{A}}\left(t_{a c}-\tau_{T}\right)}, & \text { if } \tau_{T}<t_{a c}<\tau_{R} \\ \left(P_{a b c, a a b}\left(\tau_{T}\right) P_{A}+P_{a b c, a b c}\left(\tau_{T}\right)\right) \frac{2}{\theta_{R}} \mathrm{e}^{-\frac{2}{\theta_{R}}\left(t_{a c}-\tau_{R}\right)}, & \text { if } t_{a c}>\tau_{R}\end{cases}
$$

where $P_{A}=\mathrm{e}^{-\frac{2}{\theta_{A}}\left(\tau_{R}-\tau_{T}\right)}$ is the probability that two sequences entering population $A$ at time $\tau_{T}$ do 
not coalesce before they reach time $\tau_{R}$. The expectation of $t_{a c}$ is

$$
\begin{aligned}
\mathbb{E}\left(t_{a c}\right)= & \left(\frac{1}{w_{A C}}+\frac{\theta_{A}}{2}\right)+\frac{\theta_{A} w_{A C}}{2-\theta_{A} w_{A C}}\left(\tau_{T}+\frac{\theta_{A}}{2}\right) \mathrm{e}^{-\frac{2}{\theta_{A}} \tau_{T}}-\frac{2}{2-\theta_{A} w_{A C}}\left(\tau_{T}+\frac{1}{w_{A C}}\right) \mathrm{e}^{-w_{A C} \tau_{T}} \\
& +P_{a b c, a a b}\left(\tau_{T}\right)\left[\tau_{T}+\frac{\theta_{A}}{2}+P_{A}\left(\frac{\theta_{R}}{2}-\frac{\theta_{A}}{2}\right)\right]+P_{a b c, a b c}\left(\tau_{T}\right)\left(\tau_{R}+\frac{\theta_{R}}{2}\right) .
\end{aligned}
$$

The coalescent time $t_{b c}$ has the density

$$
f\left(t_{b c}\right)= \begin{cases}P_{a b c, a b c}\left(\tau_{T}\right) \frac{2}{\theta_{T}} \mathrm{e}^{-\frac{2}{\theta_{T}}\left(t_{b c}-\tau_{T}\right)}, & \text { if } \tau_{T}<t_{b c}<\tau_{R}, \\ {\left[P_{a b c, a b c}\left(\tau_{T}\right) P_{T}+\left(1-P_{a b c, a b c}\left(\tau_{T}\right)\right)\right] \frac{2}{\theta_{R}} \mathrm{e}^{-\frac{2}{\theta_{R}}\left(t_{b c}-\tau_{R}\right)},} & \text { if } t_{b c}>\tau_{R},\end{cases}
$$

with expectation

$$
\mathbb{E}\left(t_{b c}\right)=P_{a b c, a b c}\left(\tau_{T}\right)\left[\tau_{T}+\frac{\theta_{T}}{2}+P_{T}\left(\frac{\theta_{R}}{2}-\frac{\theta_{T}}{2}\right)\right]+\left(1-P_{a b c, a b c}\left(\tau_{T}\right)\right)\left(\tau_{R}+\frac{\theta_{R}}{2}\right) .
$$

Determining the threshold value of $w_{A C}$ or $M_{A C}$ for which $\mathbb{E}\left(t_{b c}\right)>\mathbb{E}\left(t_{a c}\right)$, so that the UPGMA method is inconsistent, is not analytically tractable but the threshold can be calculated numerically through a linear search for given values of parameters $(\theta \mathrm{s}$ and $\tau \mathrm{s})$.

Majority-Vote Method with Inflow Using the Markov chain characterization of the backward process of coalescent and migration described above, the probabilities of three gene trees can be easily derived (Fig. 1b). We have

$$
\begin{aligned}
& \mathbb{P}\left(G_{1}\right)=P_{a b c, a b c}\left(\tau_{T}\right)\left(1-P_{T}+\frac{1}{3} P_{T}\right)+P_{a b c, a a b}\left(\tau_{T}\right) P_{A} \cdot \frac{1}{3}, \\
& \mathbb{P}\left(G_{2}\right)=P_{a b c, a b}\left(\tau_{T}\right)+P_{a b c, a a b}\left(\tau_{T}\right)\left(1-P_{A}+\frac{1}{3} P_{A}\right)+\frac{1}{3} P_{a b c, a b c}\left(\tau_{T}\right) P_{T}, \\
& \mathbb{P}\left(G_{3}\right)=\frac{1}{3} P_{a b c, a b c}\left(\tau_{T}\right) P_{T}+\frac{1}{3} P_{a b c, a a b}\left(\tau_{T}\right) P_{A},
\end{aligned}
$$

where $P_{A}=\mathrm{e}^{-\frac{2}{\theta_{A}}\left(\tau_{R}-\tau_{T}\right)}$. For example, gene tree $G_{1}$ occurs if sequences $b$ and $c$ coalesce first. This can only occur if there is no coalescent (between sequences $a$ and $c$ ) in the time epoch $\left(0, \tau_{T}\right)$, so that the state of the Markov chain at time $\tau_{T}$ must be either $S_{a b c}$ or $S_{a a b}$. In the former case (state $S_{a b c}$ at time $\tau_{T}$ ), sequences $b$ and $c$ may coalesce first, in either species $T$ (with probability $1-P_{T}$ ) or species $R$ (with probability $P_{T} \cdot \frac{1}{3}$ ). In the latter case (state $S_{a a b}$ at time $\tau_{T}$ ), sequences $b$ and $c$ may coalesce first only if there is no coalescence (between sequences $a$ and $c$ ) in species $A$ between $\tau_{T}$ and $\tau_{R}$ (with probability $P_{A} \cdot \frac{1}{3}$ ).

It is easy to see that $\mathbb{P}\left(G_{3}\right)<\min \left\{\mathbb{P}\left(G_{1}\right), \mathbb{P}\left(G_{2}\right)\right\}$. We have $\mathbb{P}\left(G_{1}\right)<\mathbb{P}\left(G_{2}\right)$ if and only if $P_{a b c, a b c}\left(\tau_{T}\right)\left(2-P_{T}\right)+P_{a b c, a a b}\left(\tau_{T}\right) P_{A}<1$, or if and only if

$$
\mathrm{e}^{-w_{A C} \tau_{T}}\left(2-P_{T}\right)+\frac{\theta_{A} w_{A C}\left(\mathrm{e}^{-w_{A C} \tau_{T}}-\mathrm{e}^{-\frac{2}{\theta_{A}} \tau_{T}}\right)}{2-\theta_{A} w_{A C}} P_{A}<1
$$

Again the threshold value of $w_{A C}$ for this condition to be satisfied, so that the majority-vote method is inconsistent, can be calculated numerically through a linear search. 
UPGMA Method with Outflow Similar to the IM model of Figure 1b, we use a Markov chain to characterize the coalescent-migration process in the time interval $\left(0, \tau_{T}\right)$ (Fig. $\left.1 \mathrm{~d}\right)$. The migration rate from $C$ to $A$ (in forward time) is $M_{C A}$ migrants per generation or $w_{C A}=m_{C A} / \mu=4 M_{C A} / \theta_{A}$ when time is scaled by the mutation rate. The coalescent rate (in population $C$ after sequence $a$ moves into $C)$ is $1 /\left(2 N_{C}\right)$ per generation or $2 / \theta_{C}$ on the mutational time scale. For a sample of three sequences $(a, b, c)$, the three states of the Markov chain are $S_{a b c}$, $S_{c c b}$ and $S_{c b}$, where $S_{c c b}$ indicates three sequences exist with two sequences in species $C$ and one in $B$, and $S_{c b}$ indicates two sequences exist with one in species $C$ and one in $B$. The rate matrix $Q$ is

\begin{tabular}{c|ccc} 
& $S_{a b c}$ & $S_{c c b}$ & $S_{c b}$ \\
\hline$S_{a b c}$ & $-w_{C A}$ & $w_{C A}$ & 0 \\
$S_{c c b}$ & 0 & $-\frac{2}{\theta_{C}}$ & $\frac{2}{\theta_{C}}$ \\
$S_{c b}$ & 0 & 0 & 0
\end{tabular}

This has the eigenvalues $\lambda_{1}=0, \lambda_{2}=-\frac{2}{\theta_{C}}$, and $\lambda_{3}=-w_{C A}$. The transition probability matrix $P(t)=\exp (Q t)$ is

\begin{tabular}{c|ccc} 
& $S_{a b c}$ & $S_{c c b}$ & $S_{c b}$ \\
\hline$S_{a b c}$ & $\mathrm{e}^{-w_{C A} t}$ & $\frac{\theta_{C} w_{C A}}{2-\theta_{C} w_{C A}}\left(\mathrm{e}^{-w_{C A} t}-\mathrm{e}^{-\frac{2}{\theta_{C}} t}\right)$ & $1-\frac{2}{2-\theta_{C} w_{C A}} \mathrm{e}^{-w_{C A} t}+\frac{\theta_{C} w_{C A}}{2-\theta_{C} w_{C A}} \mathrm{e}^{-\frac{2}{\theta_{C}} t}$ \\
$S_{c c b}$ & 0 & $\mathrm{e}^{-\frac{2}{\theta_{C} t}}$ & $1-\mathrm{e}^{-\frac{2}{\theta_{C}} t}$ \\
$S_{c b}$ & 0 & 0 & 1
\end{tabular}

Sequences $a$ and $c$ can coalesce in populations $C, T$ and $R$. Sequences $b$ and $c$ or $a$ and $b$ can coalesce in $T$ and $R$. The probability density of coalescent time $t_{a c}$ is

$$
f\left(t_{a c}\right)= \begin{cases}P_{a b c, c c b}\left(t_{a c}\right) \frac{2}{\theta_{C}}, & \text { if } 0<t_{a c}<\tau_{T}, \\ P_{a b c, c c b}\left(\tau_{T}\right) \frac{2}{\theta_{T}} \mathrm{e}^{-\frac{2}{\theta_{T}}\left(t_{a c}-\tau_{T}\right)}, & \text { if } \tau_{T}<t_{a c}<\tau_{R}, \\ \left(P_{a b c, c c b}\left(\tau_{T}\right) P_{T}+P_{a b c, a b c}\left(\tau_{T}\right)\right) \frac{2}{\theta_{R}} \mathrm{e}^{-\frac{2}{\theta_{R}}\left(t_{a c}-\tau_{R}\right)}, & \text { if } t_{a c}>\tau_{R},\end{cases}
$$

with expectation

$$
\begin{aligned}
\mathbb{E}\left(t_{a c}\right)= & \left(\frac{1}{w_{C A}}+\frac{\theta_{C}}{2}\right)+\frac{\theta_{C} w_{C A}}{2-\theta_{C} w_{C A}}\left(\tau_{T}+\frac{\theta_{C}}{2}\right) \mathrm{e}^{-\frac{2}{\theta_{C}} \tau_{T}}-\frac{2}{2-\theta_{C} w_{C A}}\left(\tau_{T}+\frac{1}{w_{C A}}\right) \mathrm{e}^{-w_{C A} \tau_{T}} \\
& +P_{a b c, c c b}\left(\tau_{T}\right)\left[\tau_{T}+\frac{\theta_{T}}{2}+P_{T}\left(\frac{\theta_{R}}{2}-\frac{\theta_{T}}{2}\right)\right]+P_{a b c, a b c}\left(\tau_{T}\right)\left(\tau_{R}+\frac{\theta_{R}}{2}\right) .
\end{aligned}
$$

The coalescent time $t_{b c}$ has the density

$$
f\left(t_{b c}\right)= \begin{cases}\frac{2}{\theta_{T}} \mathrm{e}^{-\frac{2}{\theta_{T}}\left(t_{b c}-\tau_{T}\right)}, & \text { if } \tau_{T}<t_{b c}<\tau_{R} \\ P_{T} \frac{2}{\theta_{R}} \mathrm{e}^{-\frac{2}{\theta_{R}}\left(t_{b c}-\tau_{R}\right)}, & \text { if } t_{b c}>\tau_{R}\end{cases}
$$

with expectation

$$
\mathbb{E}\left(t_{b c}\right)=\tau_{T}+\frac{\theta_{T}}{2}+P_{T}\left(\frac{\theta_{R}}{2}-\frac{\theta_{T}}{2}\right) .
$$


The probability density of the coalescent time $t_{a b}$ is

$$
f\left(t_{a b}\right)= \begin{cases}P_{a b c, c c b}\left(\tau_{T}\right) \frac{2}{\theta_{T}} \mathrm{e}^{-\frac{2}{\theta_{T}}\left(t_{a b}-\tau_{T}\right)}, & \text { if } \tau_{T}<t_{a b}<\tau_{R}, \\ {\left[P_{a b c, c c b}\left(\tau_{T}\right) P_{T}+\left(1-P_{a b c, c c b}\left(\tau_{T}\right)\right)\right] \frac{2}{\theta_{R}} \mathrm{e}^{-\frac{2}{\theta_{R}}\left(t_{a b}-\tau_{R}\right)},} & \text { if } t_{a b}>\tau_{R},\end{cases}
$$

with expectation

$$
\mathbb{E}\left(t_{a b}\right)=P_{a b c, c c b}\left(\tau_{T}\right)\left[\tau_{T}+\frac{\theta_{T}}{2}+P_{T}\left(\frac{\theta_{R}}{2}-\frac{\theta_{T}}{2}\right)\right]+\left(1-P_{a b c, c c b}\left(\tau_{T}\right)\right)\left(\tau_{R}+\frac{\theta_{R}}{2}\right) .
$$

It is easy to see that $\mathbb{E}\left(t_{a b}\right)>\max \left\{\mathbb{E}\left(t_{a c}\right), \mathbb{E}\left(t_{b c}\right)\right\}$. Again the threshold value of $w_{C A}$ or $M_{C A}$ for which $\mathbb{E}\left(t_{b c}\right)>\mathbb{E}\left(t_{a c}\right)$, so that the UPGMA method is inconsistent, can be calculated numerically through a linear search.

Majority-Vote Method with Outflow The procedure we used previously to analyze the IM model with inflow is also applied here (Fig. 1d). We have

$$
\begin{aligned}
& \mathbb{P}\left(G_{1}\right)=P_{a b c, a b c}\left(\tau_{T}\right)\left(1-P_{T}+\frac{1}{3} P_{T}\right)+\frac{1}{3} P_{a b c, c c b}\left(\tau_{T}\right), \\
& \mathbb{P}\left(G_{2}\right)=P_{a b c, c b}\left(\tau_{T}\right)+\frac{1}{3} P_{a b c, a b c}\left(\tau_{T}\right) P_{T}+\frac{1}{3} P_{a b c, c c b}\left(\tau_{T}\right), \\
& \mathbb{P}\left(G_{3}\right)=\frac{1}{3} P_{a b c, a b c}\left(\tau_{T}\right) P_{T}+\frac{1}{3} P_{a b c, c c b}\left(\tau_{T}\right),
\end{aligned}
$$

It is easy to see that $G_{3}$ is the least probable gene tree, with $\mathbb{P}\left(G_{3}\right)<\min \left\{\mathbb{P}\left(G_{1}\right), \mathbb{P}\left(G_{2}\right)\right\}$. Furthermore, $\mathbb{P}\left(G_{1}\right)<\mathbb{P}\left(G_{2}\right)$ if and only if $P_{a b c, a b c}\left(\tau_{T}\right)\left(1-P_{T}\right)<P_{a b c, c b}\left(\tau_{T}\right)$, or if and only if

$$
\mathrm{e}^{-w_{C A} \tau_{T}}\left(1-P_{T}\right)+\frac{2 \mathrm{e}^{-w_{C A} \tau_{T}}-\left(\theta_{C} w_{C A}\right) \mathrm{e}^{-\frac{2}{\theta_{C}} \tau_{T}}}{2-\theta_{C} w_{C A}}<1
$$

Again a linear-search algorithm can be used to determine the value of $w_{C A}$ for which this condition is satisfied and the majority-vote method is inconsistent.

\section{RESULTS}

\section{The Majority-Vote and UPGMA Methods}

Here we apply the theoretical results developed above to determine the amount of gene flow, as measured by the migration rate $M$ in the IM model and the introgression probability $\varphi$ in the MSci model, that is sufficient to mislead species tree estimation. These thresholds define the boundary of the zone of inconsistency for each gene flow model and each inference method. In Figure 2, the threshold values of $\varphi$ under the MSci model and of $M$ under the IM model are plotted against $\tau_{T} / \tau_{R}$. All populations are assumed to have the same size $(\theta)$, and $\tau_{R}=5 \theta$ or $10 \theta$ is fixed. In the case of the MSci model, there is an introgression event at time $\tau_{H}=\tau_{S}=\tau_{R} / 5$ (see Figure 1a \& c). Note that a larger $\tau_{T} / \tau_{R}$ (or $\tau_{T}$ since $\tau_{R}$ is fixed) means that the internal branch in the species tree is shorter and the species tree is more challenging to recover, so that all methods are expected to be more sensitive to gene flow.

We focus on hard species trees with a short internal branch, that is, with $\tau_{T} / \tau_{R}$ larger than $\frac{1}{2}$ or even close to 1 . Note that the plotted threshold value is the point at which the two species 
(a) MSci model, $\tau_{R}=5 \theta$

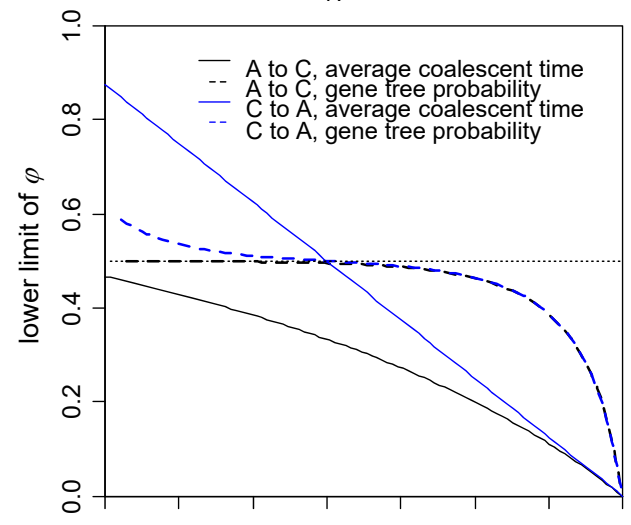

(c) MSci model, $\tau_{R}=10 \theta$

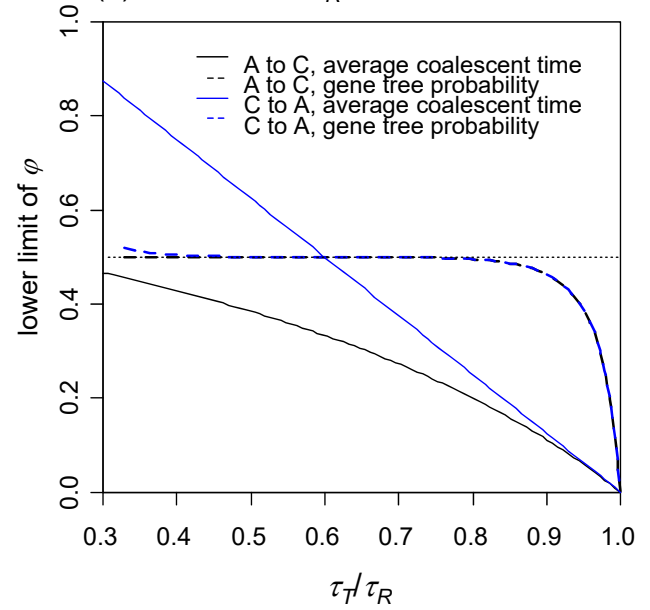

(b) IM model, $\tau_{R}=5 \theta$

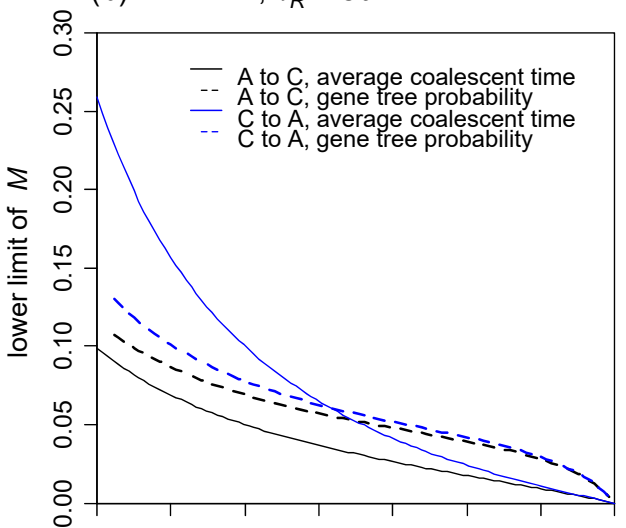

(d) IM model, $\tau_{R}=10 \theta$

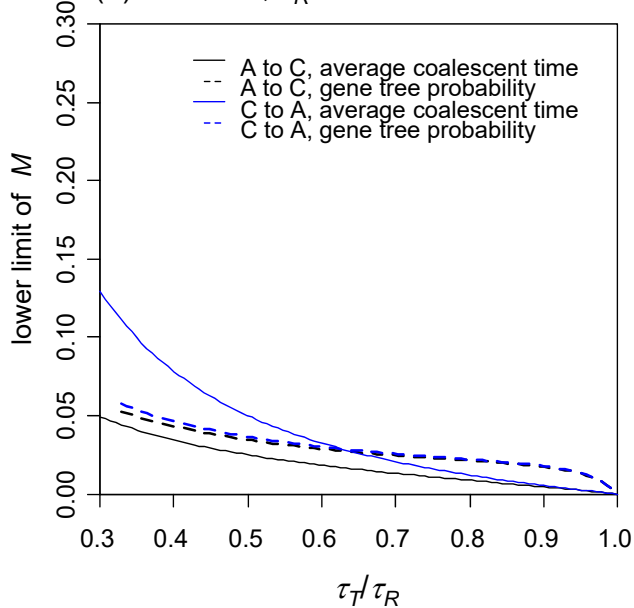

Fig. 2. The lower limit of the introgression probability $\varphi$ in the MSci model and of the migration rate $M$ in the IM model that is necessary and sufficient to mislead species tree estimation methods UPGMA (based on average coalescent times) and Majority-vote (based on gene tree probabilities), plotted against $\tau_{T} / \tau_{R}$. All populations are assumed to have the same size parameter $(\theta)$, while the age of the root is $\tau_{R}=5 \theta$ in (a) and (b) and $\tau_{R}=10 \theta$ in (c) and (d). In the MSci model, we use $\tau_{H}=\tau_{S}=\tau_{R} / 5$. Note that the $\varphi$ and $M$ limits do not depend on the precise value of $\theta$.

trees are equally good. For example, in the case of the inflow introgression model and the majority-vote method, with $\tau_{R}=5 \theta$ and $\tau_{T} / \tau_{R}=0.95$, the threshold is $\varphi_{\text {lim }}=0.282$ (Fig. 2a). Thus, if and only if the introgression probability is higher than $28.2 \%$ will the mismatching gene tree $G_{2}$ be more frequent than the matching gene tree $G_{1}$, and the majority-vote method infer the incorrect species tree (and be inconsistent). The threshold of $\varphi_{\text {lim }}=0.0588$ for the UPGMA method (Fig. 2a) is much lower, suggesting that the UPGMA method is much more sensitive to gene flow than the majority-vote method. The results for outflow introgression are similar, with the majority-vote method being more robust to gene flow than the UPGMA method. Indeed when $\tau_{T}>0.9 \tau_{R}$, the $\varphi$ thresholds are nearly identical for inflow versus outflow introgressions.

For the inflow migration model (Fig. 2b) and with $\tau_{R}=5 \theta$ and $\tau_{T} / \tau_{R}=0.95$, the lower limit for migration rate is $M_{A C}=0.0183$ for the majority-vote method and $M_{A C}=0.00473$ for UPGMA. The limiting $M_{C A}$ values for the outflow model are similar. Again the majority-vote method is much more robust to gene flow than UPGMA. Note that in population genetic models of subdivision, gene flow of rates $M \approx 0.1$ immigrants per generation is considered low enough 
so that strong population differentiation will not occur, yet such low levels can still lead to inconsistency when the species tree is difficult to reconstruct due to short internal branches.

\section{UPGMA Using Reconstructed Gene Trees}

The gene tree probabilities we derived above are for the true gene trees. In analyses of real data gene trees estimated from sequence alignments may differ from the true gene trees due to inference errors. Here we study properties of the majority-vote method when it is applied to estimated gene trees. We expect random sampling errors to be unimportant for the UPGMA method based on average sequence distances between species because the number of sites in multi-locus datasets is huge.

The impact of phylogenetic errors under the MSC model without gene flow and in the case of three species and Jukes-Cantor (JC) model (Jukes and Cantor, 1969) was studied by Yang (2002). Without phylogenetic errors, the probabilities of the gene trees satisfy $\mathbb{P}\left(G_{1}\right)>\mathbb{P}\left(G_{2}\right)=$ $\mathbb{P}\left(G_{3}\right)$ (Hudson, 1983). Let $\mathbb{P}_{n}\left(G_{k}\right), k=1,2,3$, be the probability that the estimated gene tree (the ML gene tree, for example) is $G_{k}$ at a locus with sequences of $n$ sites, with $\mathbb{P}_{\infty}\left(G_{k}\right)=\mathbb{P}\left(G_{k}\right)$. While phylogenetic reconstruction errors may cause the true matching gene tree $\left(G_{1}\right)$ to be reconstructed as a mismatching gene tree $\left(G_{2}\right.$ or $\left.G_{3}\right)$ and vice versa, reconstruction errors on balance always inflate the gene tree-species tree mismatch probability, but do not change the order of the gene trees. In other words, $\mathbb{P}_{n}\left(G_{1}\right)<\mathbb{P}\left(G_{1}\right)$ and $\mathbb{P}_{n}\left(G_{2}\right)>\mathbb{P}\left(G_{2}\right)$, but the relationship $\mathbb{P}_{n}\left(G_{1}\right)>\mathbb{P}_{n}\left(G_{2}\right)=\mathbb{P}_{n}\left(G_{3}\right)$ still holds (Yang, 2002). Thus the majority-vote method, when applied to estimated gene trees, is consistent, and the probability of inferring the correct species tree will approach one with the increase in the number of loci or gene trees, even if the gene trees are estimated with sampling errors. The internal branch length in the species tree (in coalescent units) is nevertheless inconsistently and under-estimated, because the gene tree probabilities are distorted by phylogenetic errors.

The effects of phylogenetic errors on the gene-tree probabilities when there is gene flow (under either the IM or MSci models) are unknown. Here we use simulation to explore the issue. The ML gene tree for the case of three sequences and JC model with the molecular clock is analytically tractable (Yang, 1994, 2000). The sequence alignment at each locus can be summarized as five site-pattern counts $\left(n_{0}-n_{4}\right)$, for $x x x, x x y, x y x, y x x$, and $x y z$, where $x, y, z$ are any three distinct nucleotides, and the gene trees $G_{1}, G_{2}$ or $G_{3}$ is the ML tree if $n_{1}, n_{2}$, or $n_{3}$ is the greatest among the three. There is then no need for ML iteration to estimate the gene tree and branch lengths at each locus. We used this approach to calculate the probabilities of estimated gene trees $\mathbb{P}_{n}\left(G_{1}\right), \mathbb{P}_{n}\left(G_{2}\right)$, and $\mathbb{P}_{n}\left(G_{3}\right)$ by simulating $10^{5}$ datasets or loci using BPP4 under the MSci or IM models (Yang, 2015; Flouris et al., 2018). In Figure 3 we choose a set of parameter values for the MSci and IM models from Figure $2 \mathrm{a} \& \mathrm{~b}$ for which $\mathbb{P}\left(G_{1}\right)=\mathbb{P}\left(G_{2}\right)$, and simulated data at different sequence lengths. In this case phylogenetic reconstruction errors are seen to inflate the gene tree-species tree mismatch probability, with $\mathbb{P}_{n}\left(G_{1}\right)<\mathbb{P}\left(G_{1}\right)$.

We then used a linear search to find the minimum $\varphi$ in the MSci model and minimum $M$ in the IM model at which $\mathbb{P}_{n}\left(G_{1}\right)=\mathbb{P}_{n}\left(G_{2}\right)$, with the gene tree probabilities determined by simulating $10^{5}$ loci and for each locus by determining the ML tree using the observed site pattern counts. The results for the MSci model are shown in Figure 4a\&c. We focus on hard species trees and a small amount of introgression, with $\varphi \ll \frac{1}{2}$. In such cases phylogenetic reconstruction errors inflate gene tree-species tree conflicts, with $\mathbb{P}_{n}\left(G_{1}\right)<\mathbb{P}\left(G_{1}\right)$. As a result, the low limit of $\varphi$ necessary to mislead the majority-vote method of species tree estimation is lower than when true gene trees are used (Fig. 2). In other words, species tree estimation is more sensitive to introgression when gene trees are reconstructed from sequence data than when true gene trees are used. The patterns are the same for the inflow $(A \rightarrow C)$ and outflow $(C \rightarrow A)$ introgressions.

The results under the IM model are similar (Fig. 4b\&d). For hard species trees with short internal branches, phylogenetic reconstruction errors inflate the gene tree-species tree conflicts, 

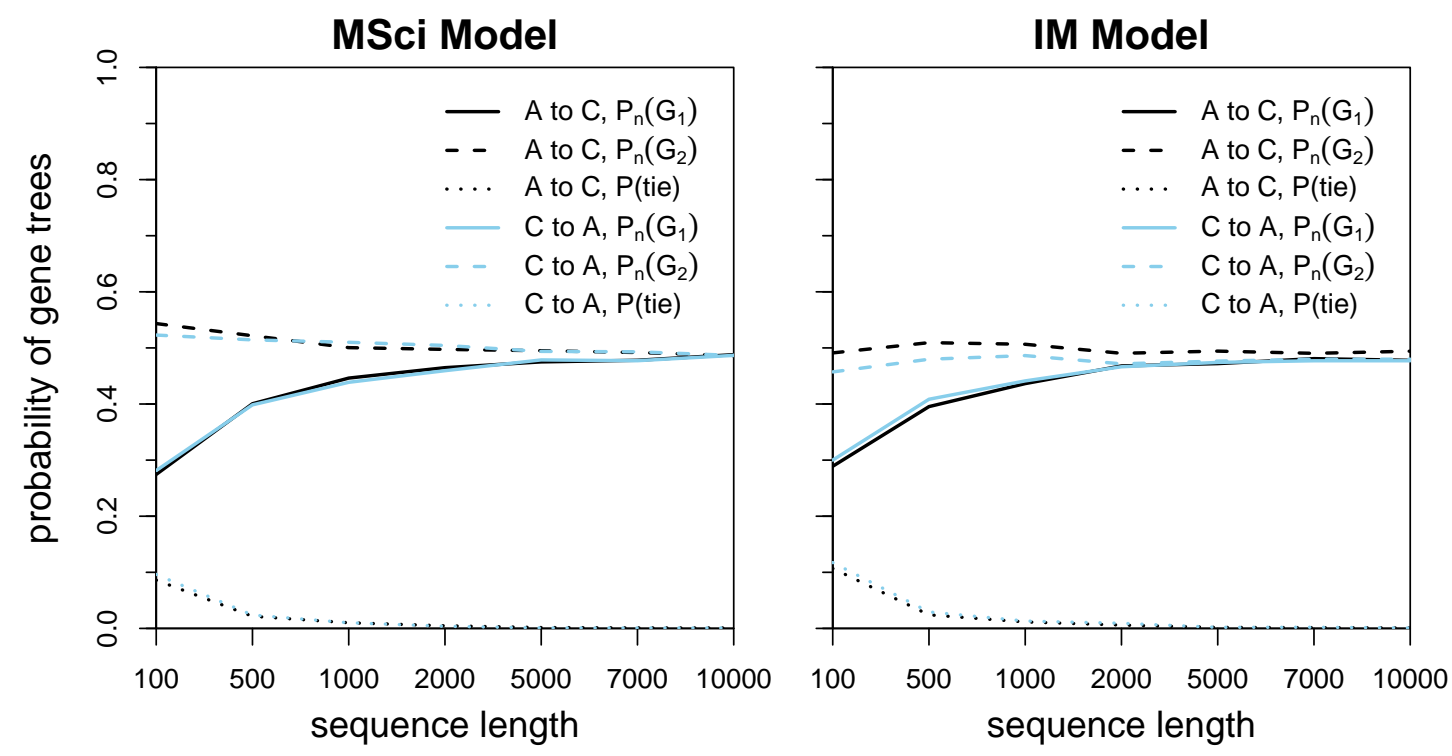

Fig. 3. Probabilities of estimated gene trees, $\mathbb{P}_{n}\left(G_{1}\right)$ and $\mathbb{P}_{n}\left(G_{2}\right)$, as a function of sequence length $(n)$, under the MSci and IM models. The following parameter values are used: $\theta=0.01$ for all populations, $\tau_{T}=0.04$, and $\tau_{R}=0.05$; and for the MSci model, $\tau_{H}=0.01$ and $\varphi=0.4638$ for inflow introgression $(A \rightarrow C)$ and 0.4643 for outflow introgression $(C \rightarrow A)$, while for the IM model, $M=0.0393$ for inflow migration and 0.0419 for outflow immigration. At those parameter values, $\mathbb{P}\left(G_{1}\right)=\mathbb{P}\left(G_{2}\right)$ when the sequence length is infinity (so that there are no phylogenetic reconstruction errors). $\mathbb{P}($ tie) is the proportion of datasets in which two or three gene trees are equally best.

making the estimation of the species tree even harder.

\section{Full-Likelihood Methods}

Full-likelihood methods applied to multi-locus sequence alignments, including ML and Bayesian methods, integrate over the gene tree topologies and coalescent times and naturally accommodate phylogenetic reconstruction errors due to limited number of sites at each locus (see, for a review, Xu and Yang, 2016). They are not tractable analytically. Nevertheless, in the case of three species and three sequences per locus, an efficient ML implementation of the MSC model exists in the 3S program (Yang, 2002; Dalquen et al., 2017). Here we use 3s to analyze datasets of 10,000 loci (with sequence length $n=1000$ ), assuming that at such large data size, the estimates are close to the infinite-data limits. In Figure 5, we conducted similar calculations to Figure 4, but with ML (the 3s program) replacing majority-vote. Consider 5a, where the true model is the MSci model. For each value of $\tau_{T}$, a linear search (bisection) is used to find the lowest value of $\varphi$ in the true MSci model at which the two species trees have the same log likelihood under the MSC model, $\ell_{1}=\ell_{2}$, where the log likelihood is calculated under the JC model and the molecular clock by averaging over the three gene trees and integrating over the two coalescent times in each gene tree (Yang, 2002). For each $\varphi$ (and $\tau_{T}$ as well as other parameters in the MSci model), a dataset of 10,000 loci is simulated and analyzed using $3 \mathrm{~s}$ to determine whether $\ell_{1}>\ell_{2}$ (that is, whether $\varphi$ is too small). Each round of bisection reduces the interval of uncertainty by a half. The scatter-points in the plots (Fig. 5) show some fluctuations, due to the finite nature of the datasets, and are used to fit a smoothed curve.

The lower limits of $\varphi$ in the MSci model and of $M$ in the IM model for the ML/3S method 

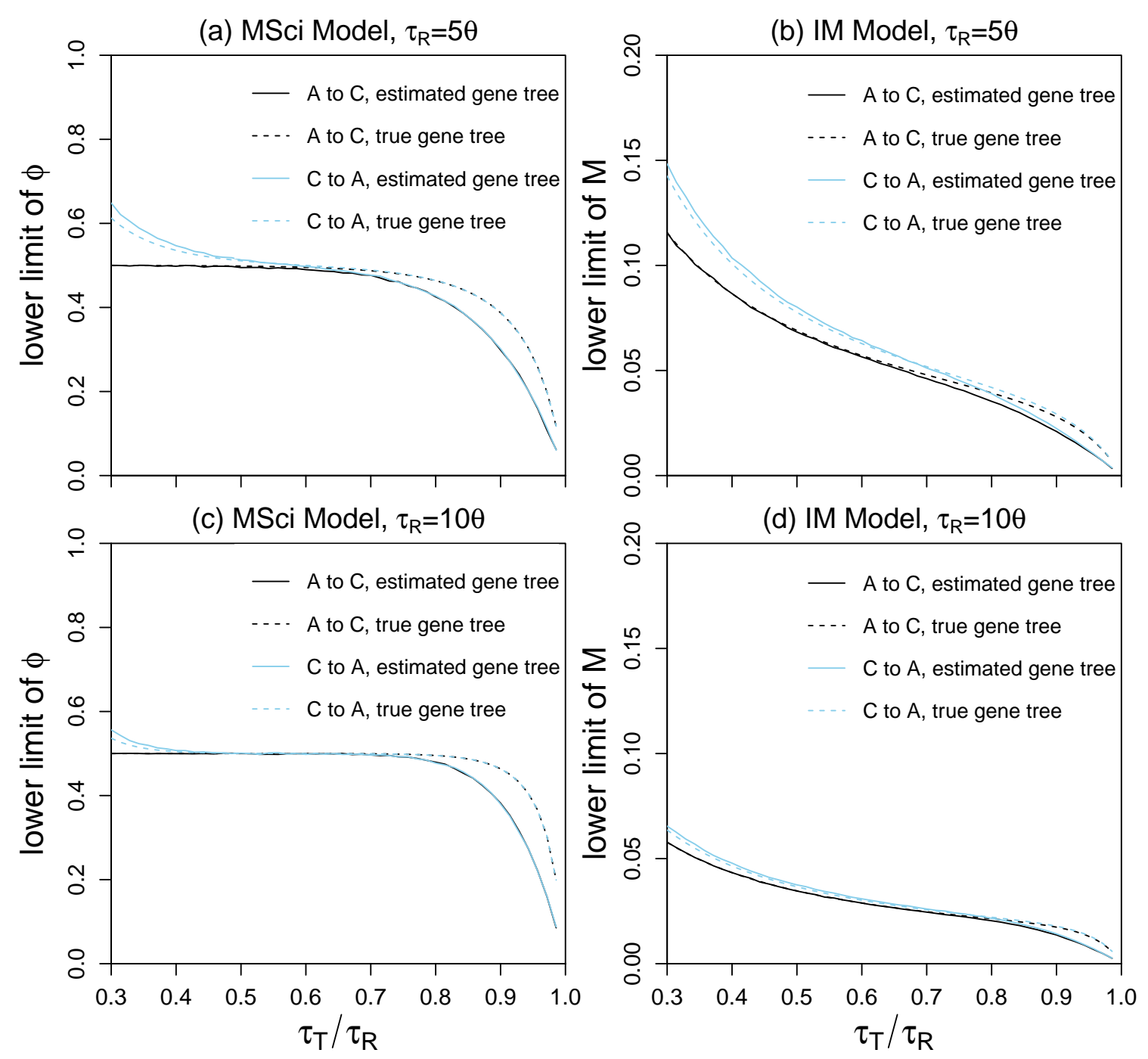

Fig. 4. The lower limit of the introgression probability $\varphi$ in the MSci model and the migration rate $M$ in the IM model necessary to mislead the majority-vote method of species tree estimation, plotted against $\tau_{T} / \tau_{R}$, when either the true or estimated gene trees are used. For estimated gene trees the sequence length is $n=1000$. At the $\varphi$ or $M$ values shown, $\mathbb{P}\left(G_{1}\right)=\mathbb{P}\left(G_{2}\right)$ when the true gene trees are used or $\mathbb{P}_{n}\left(G_{1}\right)=\mathbb{P}_{n}\left(G_{2}\right)$ when estimated gene trees are used. The estimated gene tree is the ML tree from the sequence alignment of $n=1000$ sites, determined by using the site pattern counts at the locus. Monte Carlo simulation, with $10^{5}$ replicates (loci or sequence alignments), was used to estimate the probabilities of the ML gene trees: $\mathbb{P}_{n}\left(G_{1}\right)$ and $\mathbb{P}_{n}\left(G_{2}\right)$. Parameters other than $\varphi$ or $M$ are fixed at $\theta=0.01$ for all populations, $\tau_{H}=\tau_{S}=\tau_{R} / 5$ for the MSci model (a and c), while $\tau_{R}=5 \theta$ in (a) and (b) and $\tau_{R}=10 \theta$ in (c) and (d). Results for the true gene trees are from Figure 2, shown here for comparison.

of Figure 5 are much lower than the corresponding values for the majority-vote and the UPGMA methods of Figures 2 and 4. The ML/3s method assuming MSC without gene flow infers the incorrect species tree at much lower levels of gene flow (and is less robust to gene flow) than the majority-vote or UPGMA methods. 
(a) MSci model, $\mathrm{T}_{\mathrm{R}}=5 \theta$

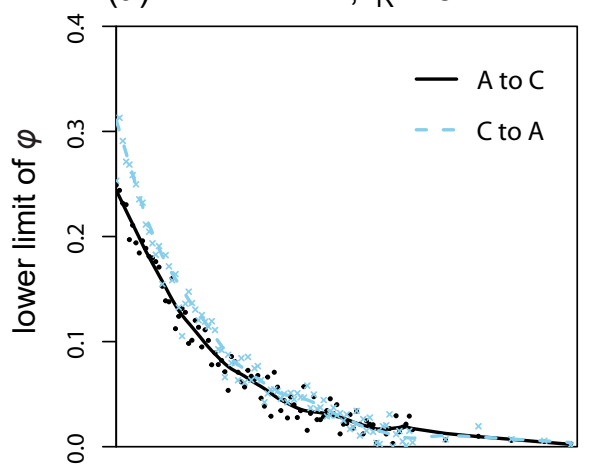

(c) MSci model, $\mathrm{T}_{\mathrm{R}}=10 \theta$

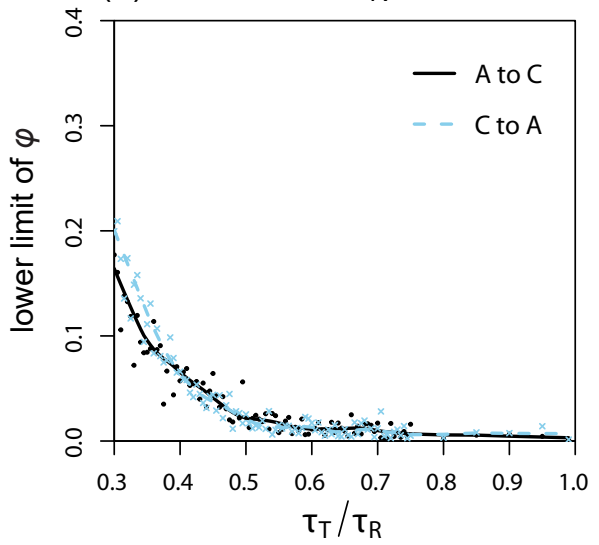

(b) IM model, $\mathrm{T}_{\mathrm{R}}=5 \theta$

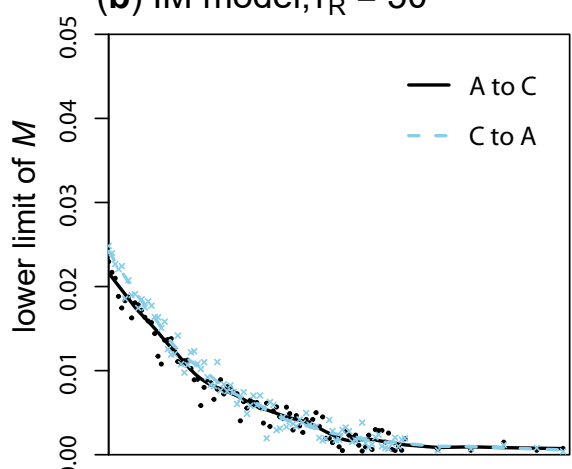

(d) IM model, $\mathrm{T}_{\mathrm{R}}=100$

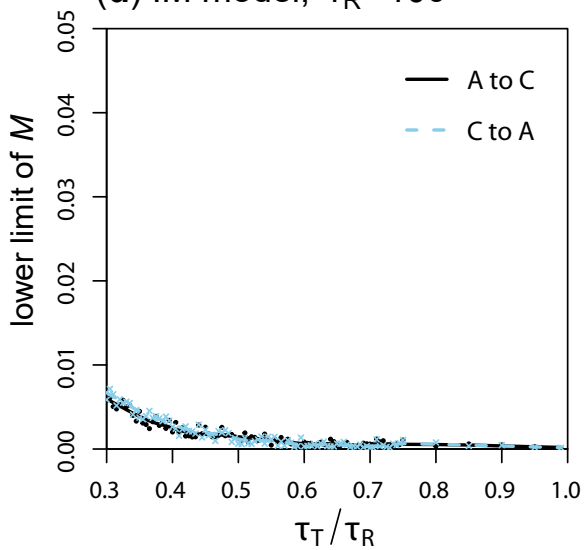

Fig. 5. The lower limit of $\varphi$ in the MSci model and $M$ in the IM model necessary to mislead the ML/3s method of species tree estimation under the MSC, plotted against $\tau_{T} / \tau_{R}$. The sequence length is 1000 . Data of 10,000 loci were analyzed using $3 \mathrm{~S}$ to determine the ML species tree. Parameter values used are the same as in Figure 4.

\section{Differences Between the IM and MSci Models}

The MSci and IM models are two idealized models that accommodate gene flow between species. The MSci model assumes episodic introgression or hybridization events that occur at fixed time points in the past, while the IM model assumes continuous-time migration with migrants occurring at a certain rate in every generation. The MSci and IM models represent two extremes and in reality a combination of the two processes may be more realistic. When the two models are applied to the same data, a frequently asked question is how the important parameters of the two models ( $\varphi$ in MSci and $M$ in IM models, say) correspond to each other. One may expect a higher migration rate $M$ should correspond to a larger introgression probability $\varphi$, but the precise relationship may depend on the values of other parameters in the models, as well as the data configurations (the number of loci, the number of sequences per locus, and the sequence length). Bearing in mind those caveats we conducted three analyses to address this question.

Firstly, we may compare the limiting values of $\varphi$ under the MSci model and $M$ in the IM model for the same $\tau_{T} / \tau_{R}$ ratio in Figure 2. For example, for the inflow migration or introgression $(A \rightarrow C)$, with $\tau_{R}=5 \theta$ and $\tau_{T} / \tau_{R}=0.95$, the limiting values to achieve equal gene tree probabilities, $\mathbb{P}\left(G_{1}\right)=\mathbb{P}\left(G_{2}\right)$, are $\varphi_{\text {lim }}=0.282$ for the MSci model and $M_{A C}=0.0183$ for the IM model. The limiting values for achieving equal average coalescent times, $\mathbb{E}\left(t_{b c}\right)=\mathbb{E}\left(t_{a c}\right)$, are $\varphi_{\lim }=0.0588$ for the MSci model and $M_{A C}=0.00473$ for the IM model. Here we are matching 
certain summaries of the data to establish a correspondence between $\varphi$ and $M$ in the two models.

Secondly, we used the IM model to simulate large datasets of 10,000 loci, with two sequences per species per locus and with sequence length of 100 or 1000 sites, and then used BPP to analyze the data under the MSci model (Flouris et al., 2019). The rationale is that the datasets are so large that random sampling errors in the estimates are negligible, as are the impact of the prior or the differences between MLEs and Bayesian estimates. In other words, the posterior means of parameters should be close to the pseudo-true parameter values, which are the limits of the MLEs when the data size approaches infinity. This approach also allows us to examine the estimates of other parameters in the model besides the rate of gene flow. The results are listed in table 1.

In the case of inflow migration $(A \rightarrow C)$, population sizes $(\theta)$ for most populations (in particular, the extant species) are well estimated, especially at $n=1000$. Species divergence times $\tau_{R}$ and $\tau_{T}$ estimated under MSci are very similar to the true values under the IM model ( 0.05 and 0.04 , respectively). The age of the hybridization node $\tau_{H}\left(=\tau_{S}\right)$ is very small, especially at the larger sequence length. Under the IM model, migration occurs over the whole time interval $\left(0, \tau_{T}\right)$, and one might naively expect $\tau_{H}$ under MSci to be close to the mid value. Instead the MSci estimate of $\tau_{H}$ is near the lower limit. Indeed the estimate should be smaller for longer sequences and/or more loci, because under the MSci model, the hybridization time must be smaller than the between-species sequence divergence time, with $\tau_{H}<t_{a c}$. Thus, longer sequences or more loci should provide stronger evidence that the minimum sequence divergence $t_{a c}$ generated under the IM model can be arbitrarily small, leading to reduced estimates of $\tau_{H}$ under the MSci model. The migration rate $M_{A C}=0.0393$ in the IM model is relatively small, while the estimates of $\varphi$ are substantial, at 33-44\%. The results for the case of outflow migration $(C \rightarrow A)$ are similar to those of inflow migration (table 1). Again, species divergence times $\tau_{R}$ and $\tau_{T}$ are well estimated, as are population sizes, even though the model is incorrect. The estimated hybridization time $\tau_{H}\left(=\tau_{S}\right)$ is very small. Although the migration rate is only $M_{A C}=0.0419$, the estimates of $\varphi$ in the MSci model are substantial, at 30-44\%. Overall, very low migration rates, on the order of $M=1-5 \%$ may correspond to substantial introgression probabilities close to $50 \%$.

Thirdly, we analyzed the case of two species $(A$ and $B)$ with one sequence per locus for each species when the true sequence distance (or the coalescent time between the two sequences) is known (Fig. 6a\&b). Using the theory for the IM model developed earlier, we have the probability density of coalescent time $t$ between the two sequences to be

$$
f_{M}(t)= \begin{cases}\frac{2 w}{2-\theta_{A} w}\left(\mathrm{e}^{-w t}-\mathrm{e}^{-\frac{2}{\theta_{A}} t}\right), & \text { if } 0<t<\tau_{R}, \\ \left(\frac{2}{2-\theta_{A} w} \mathrm{e}^{-w \tau_{R}}-\frac{\theta_{A} w}{2-\theta_{A} w} \mathrm{e}^{-\frac{2}{\theta_{A}} \tau_{R}}\right) \frac{2}{\theta_{R}} \mathrm{e}^{-\frac{2}{\theta_{R}}\left(t-\tau_{R}\right)}, & \text { if } t>\tau_{R},\end{cases}
$$

where $w=m_{A B} / \mu=4 M_{A B} / \theta_{B}$ is the mutation-scaled migration rate from $A$ to $B$. The density depends on $w$ but not on $M_{A B}$ and $\theta_{B}$ individually, so that the parameters specifying the density for the migration model are $\boldsymbol{\theta}^{(M)}=\left\{w, \theta_{A}, \theta_{R}, \tau_{R}\right\}$. Similarly the density under the introgression model is specified by parameters $\boldsymbol{\theta}^{(I)}=\left\{\varphi, \theta_{S}, \theta_{R}, \tau_{H}, \tau_{R}\right\}$ and is

$$
f_{I}(t)= \begin{cases}\varphi \frac{2}{\theta_{S}} \mathrm{e}^{-\frac{2}{\theta_{S}}\left(t-\tau_{S}\right)}, & \text { if } \tau_{S}<t<\tau_{R}, \\ {\left[\varphi \mathrm{e}^{-\frac{2}{\theta_{S}}\left(\tau_{R}-\tau_{S}\right)}+(1-\varphi)\right] \frac{2}{\theta_{R}} \mathrm{e}^{-\frac{2}{\theta_{R}}\left(t-\tau_{R}\right)},} & \text { if } t>\tau_{R},\end{cases}
$$

Note that in the MSci model (Fig. 6b), $\tau_{H}=\tau_{S}$ so we use the two interchangeably. Some parameters (such as $\tau_{R}$ and $\theta_{R}$ ) are common between the two models but they may take different values: when the parameter definition may be unclear from the context, we use $\tau_{R}^{(\mathrm{M})}$, with the superscript ' $(M)$ ' or ' $(I)$ ' to indicate the model involved. 
Table 1. Posterior means and 95\% highest probability density (HPD) credible intervals (CIs, below) for parameters in the MSci model when large datasets of 10,000 loci simulated under the IM model are analyzed using BPP under the MSci model

\begin{tabular}{|c|c|c|c|c|c|c|c|c|c|c|}
\hline$\theta_{A}$ & $\theta_{B}$ & $\theta_{C}$ & $\theta_{R}$ & $\theta_{T}$ & $\theta_{S}$ & $\theta_{H}$ & $\tau_{R}$ & $\tau_{T}$ & $\tau_{H}$ & $\varphi$ \\
\hline \multicolumn{11}{|c|}{ Inflow migration $(A \rightarrow C)$ with $M_{A C}=0.0393, n=100$ sites } \\
\hline 0.0096 & 0.0095 & 0.0111 & 0.0121 & 0.0185 & 0.0149 & 0.0106 & 0.0478 & 0.0386 & 0.0075 & 0.3319 \\
\hline 0.0093 & 0.0093 & 0.0107 & 0.0109 & 0.0131 & 0.0140 & 0.0092 & 0.0471 & 0.0372 & 0.0072 & 0.3208 \\
\hline 0.0099 & 0.0098 & 0.0115 & 0.0134 & 0.0241 & 0.0157 & 0.0119 & 0.0485 & 0.0401 & 0.0077 & 0.3435 \\
\hline \multicolumn{11}{|c|}{ Inflow migration $(A \rightarrow C)$ with $M_{A C}=0.0393, n=1000$ sites } \\
\hline 0.0091 & 0.0099 & 0.0100 & 0.0106 & 0.0115 & 0.0250 & 0.0100 & 0.0496 & 0.0398 & 0.0050 & 0.4393 \\
\hline 0.0089 & 0.0097 & 0.0098 & 0.0102 & 0.0107 & 0.0243 & 0.0095 & 0.0495 & 0.0395 & 0.0049 & 0.4300 \\
\hline 0.0094 & 0.0101 & 0.0103 & 0.0109 & 0.0122 & 0.0256 & 0.0106 & 0.0498 & 0.0400 & 0.0051 & 0.4485 \\
\hline \multicolumn{11}{|c|}{ Outflow migration $(C \rightarrow A)$ with $M_{C A}=0.0419, n=100$ sites } \\
\hline 0.0108 & 0.0098 & 0.0096 & 0.0154 & 0.0032 & 0.0122 & 0.0094 & 0.0437 & 0.0417 & 0.0068 & 0.3012 \\
\hline 0.0104 & 0.0095 & 0.0092 & 0.0145 & 0.0020 & 0.0115 & 0.0083 & 0.0431 & 0.0410 & 0.0066 & 0.2907 \\
\hline 0.0112 & 0.0101 & 0.0099 & 0.0163 & 0.0049 & 0.0129 & 0.0106 & 0.0442 & 0.0424 & 0.0071 & 0.3122 \\
\hline \multicolumn{11}{|c|}{ Outflow migration $(C \rightarrow A)$ with $M_{C A}=0.0419, n=1000$ sites } \\
\hline 0.0102 & 0.0099 & 0.0094 & 0.0111 & 0.0094 & 0.0243 & 0.0098 & 0.0485 & 0.0401 & 0.0051 & 0.4437 \\
\hline 0.0099 & 0.0097 & 0.0091 & 0.0107 & 0.0089 & 0.0237 & 0.0093 & 0.0483 & 0.0399 & 0.0050 & 0.4342 \\
\hline 0.0105 & 0.0101 & 0.0096 & 0.0115 & 0.0099 & 0.0250 & 0.0104 & 0.0488 & 0.0403 & 0.0052 & 0.4533 \\
\hline
\end{tabular}

Note. - The true model is IM, with parameter values $\theta_{A}=\theta_{B}=\theta_{C}=\theta_{T}=\theta_{R}=0.01, \tau_{R}=0.05$ and $\tau_{T}=0.04$, and with $M_{A C}=0.0393$ and $M_{C A}=0.0419$. These are the lower limits of $M$ in the IM model, at which $\mathbb{P}\left(G_{1}\right)=\mathbb{P}\left(G_{2}\right)$ (Fig. 2). Large datasets of 10,000 loci, with two sequences from each species per locus and with the sequence length $n=100$ or 1000 sites, were simulated under the IM model, and analyzed using B PP under the MSci model. Inverse-gamma priors are assigned on $\theta$ and $\tau_{0}: \theta \sim \operatorname{IG}(3,0.02)$ with mean $0.02 /(3-1)=0.01$ for all $\theta \mathrm{s}$, and $\tau_{0} \sim \operatorname{IG}(3,0.1)$ with mean 0.05 for the age of the species-tree root, while the introgression probability $\varphi$ is assigned the $\mathbb{U}(0,1)$ prior.

The Kullback-Leibler (KL) divergence

$$
D\left(\boldsymbol{\theta}^{(M)} \| \boldsymbol{\theta}^{(I)}\right)=\int_{0}^{\infty} f_{M}(t) \log \frac{f_{M}(t)}{f_{I}(t)} \mathrm{d} t
$$

is a measure of distance from the fitting introgression model to the true migration model. By minimizing $D$, we obtain the pseudo-true parameter values under MSci, $\boldsymbol{\theta}^{(I) *}$, when the true model is the IM model with parameters $\boldsymbol{\theta}^{(M)}$. Here $\boldsymbol{\theta}^{(M)}$ are fixed while $\boldsymbol{\theta}^{(I)}$ are being optimized. Because the IM model allows arbitrarily small coalescent time $t$ while $\tau_{H}>t$ under the MSci model, we have $\tau_{H}^{*}=0$. As the integrand of equation 1.32 has one or two discontinuity points, depending on whether $\tau_{R}^{(M)}$ and $\tau_{R}^{(I)}$ are identical (Fig. 7), we applied Gaussian quadrature, with 64 points, to each continuous segment to calculate the integral of equation 1.32. The BFGS optimization routine in PAML (Yang, 2007) is used to produce the MLEs. One such model fitting is illustrated in Figure 7. 
(a) IM mode

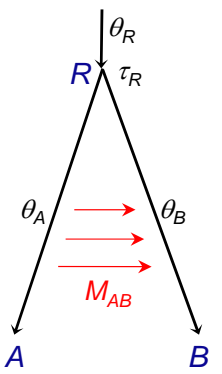

(b) MSci model

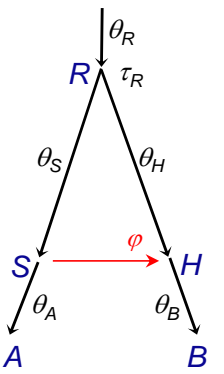

(c) MLEs under MSci

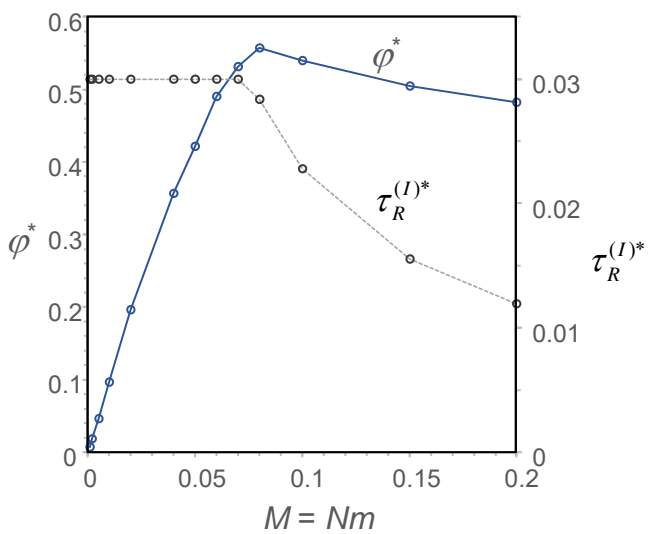

Fig. 6. The (a) migration and (b) introgression models for two species $(A, B)$, and (c) the pseudo-true parameter values of $\varphi$ and $\tau_{R}^{(I)}$ in the introgression model plotted against the migration rate $M_{A B}\left(=N m_{A B}=w \theta_{B} / 4\right)$. The pseudo-true parameter values are the parameter values under the introgression model $\boldsymbol{\theta}^{(I)}$ that minimizes the KL divergence of equation 1.32: they are the limiting values of the MLEs of $\boldsymbol{\theta}^{(I)}$, when the number of loci $L \rightarrow \infty$, when the MSci model is fitted to data of $L$ loci generated under the IM model, with two sequences (one from each species) of infinite length per locus. Other parameters in the migration model are fixed at $\tau_{R}^{(M)}=0.03$ and $\theta=0.01$ (for all populations). In the MSci model, $\theta=0.01$ is assumed for all populations, while $\varphi$ and $\tau_{R}^{(I)}$ are estimated by minimizing the KL divergence (equation 1.32).

(a)

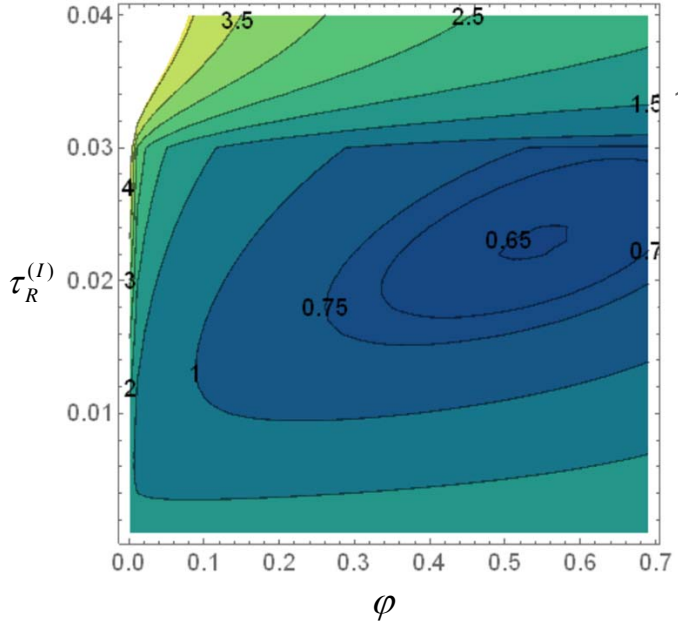

(b)

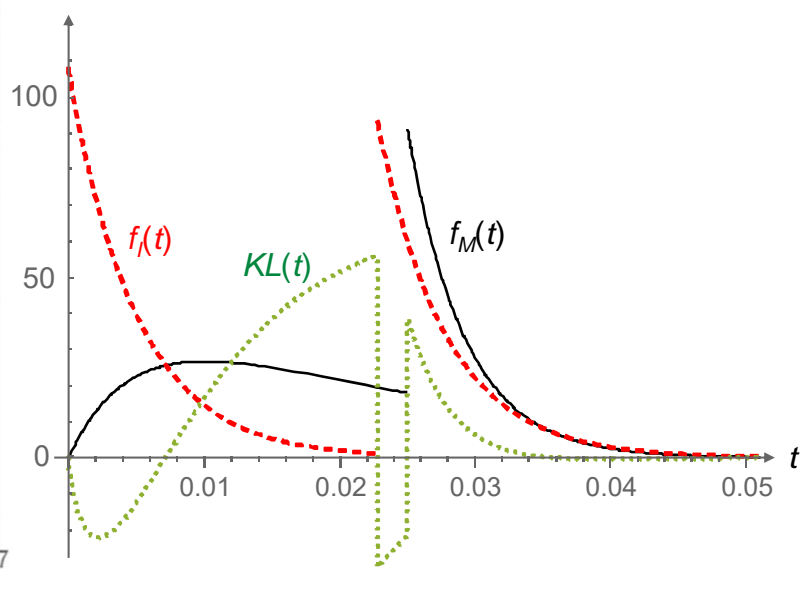

Fig. 7. (a) Contour plot of $D\left(\boldsymbol{\theta}_{M} \| \boldsymbol{\theta}_{I}\right)$ (equation 1.32) as a function of $\varphi$ and $\tau_{R}^{(I)}$ in the MSci model for the case of $M=0.1$ or $w=40$. Parameters in the IM model are $\tau_{R}^{(M)}=0.03$ and $\theta=0.01$ for all populations. The MLE under the MSci model, which minimizes $D$, is at $\varphi^{*}=0.5395$ and $\tau_{R}^{(I) *}=0.02280$. Note that in the MSci model, $\theta=0.01$ is fixed for all populations and only $\varphi$ and $\tau_{R}^{(I)}$ are optimized as $\tau_{H}=0$. The $D$ surface is not smooth at $\tau_{R}^{(I)}=\tau_{R}^{(M)}=0.03$. (b) The densities $f_{M}(t)$ (equation 1.30) and $f_{I}(t)$ (equation 1.31) as well as the integrand, $f_{M}(t) \log \frac{f_{M}(t)}{f_{I}(t)}$, of equation 1.32, plotted at the MLEs of (a). In other words, the red dashed curve $f_{I}(t)$ is the best fit of the MSci model to the infinite-sized data under the IM model represented by the black solid curve $f_{M}(t)$. Note that $f_{M}(t)$ and $f_{I}(t)$ have one discontinuity point, while the integrand has two.

Figure $6 \mathrm{c}$ shows the estimates of $\varphi$ and $\tau_{R}^{(I)}$ under the assumption that $\theta$ is the same for all 
populations and also between the two models; thus $\theta=0.01$ is fixed and only $\varphi$ and $\tau_{R}^{(I)}$ are estimated by minimizing $D$ in equation 1.32. When $M$ in the IM model is small, $\varphi$ increases nearly linearly with the increase of $M$ (Fig. 6c). However, when $M$ is large ( $>0.08$, say, corresponding to $\varphi=0.56$ ) and further increases, $\varphi$ decreases.

As a summary of all three analyses above, we note that small values of the migration rate, in the order of 0.01-0.1 migrants per generation, may correspond to large introgression probabilities and have a large effect on the genetic history of species divergences represented by the gene tree probabilities. Furthermore, the IM and MSci models make very different predictions of the distribution of coalescent times, so that the two models may be easily distinguishable using genomic sequence data when both models are implemented in the same program.

\section{DISCUSSION}

\section{The Impact of Gene Flow on Species Tree Estimation}

The impact of gene flow, either in the form of episodic introgressive hybridisation or continuous migration, on species tree estimation clearly depends on how challenging the species tree is. Our analyses suggest that when the species tree is hard with very short internal branch lengths, even a small amount of gene flow can cause species tree estimation to become inconsistent. We found that the limiting $\varphi$ and $M$ values for the gene tree probabilities are much higher than the values for the sequence distances (Figures 2 and 4), indicating that the majority-vote method based on gene tree topologies is more robust to gene flow than the UPGMA method based on average sequence distances. The full likelihood method making use of information in both gene tree topologies and branch lengths is even more sensitive. This difference in sensitivity may be explained by the fact that a small amount of gene flow may easily affect the branch lengths or sequence distances but may not alter the distribution of gene tree topologies. For hard species trees, phylogenetic reconstruction errors tend to inflate the gene tree-species tree conflicts, adding further challenges to correct inference of the species tree.

When the effects of gene flow is a concern, it is important to use species tree methods that account for both the coalescent process and cross-species gene low. Such methods are under active development. A number of methods have been developed to detect gene flow using sequence data (Green et al., 2010; Durand et al., 2011; Solis-Lemus and Ane, 2016; Dalquen et al., 2017), and furthermore, a number of methods have been developed to infer the species tree with reticulation events, including summary methods based on estimated gene tree topologies ( $\mathrm{Yu}$ et al., 2014; Yu and Nakhleh, 2015; Solis-Lemus and Ane, 2016; Wen et al., 2016; Allman et al., 2019) and full-likelihood methods applied to sequence alignments (Hey et al., 2018; Wen and Nakhleh, 2018; Zhang et al., 2018). Furthermore, we have in this paper examined the effects of gene flow on the inference of species tree topology only. The impact of gene flow on evolutionary parameters such as species divergence times and ancestral population sizes merits detailed study (Dalquen et al., 2017; Wen and Nakhleh, 2018).

\section{Introgression in the Anopheles gambiae species complex}

The Anopheles gambiae species complex is comprised of eight recognized species and includes major malaria vectors in Africa. Genome sequence data from six of the species, A. gambiae $(\mathrm{G})$, A. coluzzii (C), A. arabiensis (A), A. melas (L), A. merus (R), and A. quadriannulatus (Q), have been analyzed to estimate the species phylogeny and to infer the direction and intensity of gene

flow across species (Fontaine et al., 2015; Thawornwattana et al., 2018). A. gambiae, A. coluzzii, and $A$. arabiensis have large overlapping geographical distributions across sub-Saharan Africa and are major malaria vectors (Wiebe et al., 2017). A. melas and A. merus are found in coastal waters of eastern and western Africa, respectively, and are minor vectors. A. quadriannulatus 


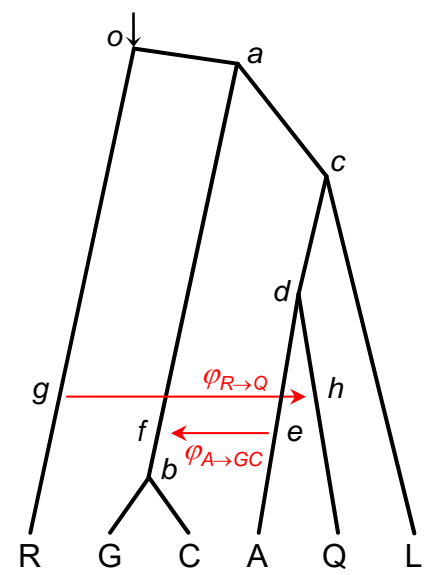

Fig. 8. The species tree for the Anopheles gambiae species complex inferred by Thawornwattana et al. (2018) from the Xag region of the X chromosome, with two migration events for the autosomes. Redrawn following (Thawornwattana et al., 2018, Fig. 6).

does not bite humans and is not a malaria vector.

Fontaine et al. (2015) analyzed the genomic sequence data for the six species using sliding windows and suggested that gene flow is so widespread that the predominant gene trees for the autosomes are different from the species phylogeny, and that the $\mathrm{X}$ chromosome, which is apparently not affected by gene flow, reflects the true species history. This conclusion is supported in the re-analysis of Thawornwattana et al. (2018), although the inferred species trees were different. The species tree obtained by Thawornwattana et al. (2018) for the $\mathrm{X}$-chromosomal data is shown in Figure 8. Besides being inferred using coalescent-based methods that accommodate deep coalescence, this tree is supported by additional sources of evidence, such as chromosome inversion data and well-known evidence of introgression from A. arabiensis $\rightarrow$ A. gambiae + A. coluzzii (Slotman et al., 2005). Moreover, computer simulation confirmed that the species trees inferred by Fontaine et al. (2015) were artefactual and reflected systematic errors of the sliding-windows analyses (Thawornwattana et al., 2018). Here we apply the theory of this paper to the species tree of Figure 8 and the parameter estimates under MSci from Flouris et al. (2019, table 1) to confirm that the introgression rates affecting the autosomes are high enough to mislead species tree estimation.

For the $\mathrm{A} \rightarrow \mathrm{G}$ introgression, we used parameter estimates ( $\tau \mathrm{s}$ and $\theta \mathrm{s}$ ) for the triplet AQG to determine the low limit of $\varphi$ in the MSci model. We retrieved the BPP estimates of parameters $\tau_{\mathrm{s}}$ and $\theta$ s for nodes $a, d, e$, and $f$ in the species tree of Figure 8 from Flouris et al. $(2019$, table 1$)$ to calculate $\varphi_{\text {lim }}$ for outflow (equations 1.14 and 1.16). The results are shown in table 2. For both the coding and noncoding data and for almost all chromosomal arms, the estimates $(\hat{\varphi})$ are larger than the limiting values $\left(\varphi_{\mathrm{lim}}\right)$. Thus, both majority-vote (based on gene tree topologies) and UPGMA (based on average sequence divergences) are inconsistent and are expected to infer an incorrect species tree.

For the $\mathrm{R} \rightarrow \mathrm{Q}$ introgression, we used the RQA triplet and the inflow equations 1.5 and 1.7. All estimates of $\varphi_{R \rightarrow Q}$ are smaller than $\varphi_{\text {lim }}$ for both the majority-vote and UPGMA methods except for the $3 \mathrm{La}$ coding region (table 2). Thus, for most of the autosomal arms introgression from $\mathrm{R}$ to $\mathrm{Q}$ did not reach a sufficient intensity to mislead species tree estimation.

\section{Migration, introgression, and the concept of species tree}

The concept of the true species tree in the presence of cross-species gene flow may be poorly defined, especially if the models of gene flow are too simple to capture the major features 
Table 2. Estimates and limiting values of $\varphi$ for different chromosomal arms obtained from the genomic data of the Anopheles gambiae species complex

\begin{tabular}{|c|c|c|c|c|c|c|}
\hline \multirow[b]{2}{*}{ Dataset } & \multicolumn{3}{|c|}{$A \rightarrow G$} & \multicolumn{3}{|c|}{$R \rightarrow Q$} \\
\hline & $\hat{\varphi}_{A \rightarrow G}$ & $\varphi_{\lim }(\mathrm{UPGMA})$ & $\varphi_{\lim }(\mathrm{M}-\mathrm{V})$ & $\hat{\varphi}_{R \rightarrow Q}$ & $\varphi_{\lim }(\mathrm{UPGMA})$ & $\varphi_{\lim }(\mathrm{M}-\mathrm{V})$ \\
\hline $2 \mathrm{~L} 1+2$ coding & 0.94 & 0.89 & 0.74 & 0.28 & 0.77 & 0.76 \\
\hline $2 \mathrm{~L} 1+2$ noncoding & 0.98 & 0.60 & 0.56 & 0.00 & 0.25 & 0.35 \\
\hline 2La coding & 0.73 & 0.14 & 0.16 & 0.01 & 0.30 & 0.42 \\
\hline 2La noncoding & 0.64 & 0.00 & 0.00 & 0.00 & 0.18 & 0.29 \\
\hline 2R coding & 0.97 & 0.90 & 0.81 & 0.34 & 0.80 & 0.79 \\
\hline 2R noncoding & 0.97 & 0.68 & 0.63 & 0.22 & 0.82 & 0.80 \\
\hline $3 \mathrm{~L} 1+2$ coding & 0.94 & 0.76 & 0.66 & 0.32 & 0.70 & 0.69 \\
\hline $3 \mathrm{~L} 1+2$ noncoding & 0.96 & 0.73 & 0.64 & 0.33 & 0.83 & 0.82 \\
\hline 3La coding & 0.93 & 0.87 & 0.78 & 0.65 & 0.56 & 0.56 \\
\hline 3La noncoding & 0.98 & 0.77 & 0.66 & 0.54 & 0.57 & 0.55 \\
\hline 3R coding & 0.95 & 0.98 & 0.95 & 0.43 & 0.65 & 0.64 \\
\hline 3R noncoding & 0.98 & 0.64 & 0.61 & 0.03 & 0.30 & 0.38 \\
\hline
\end{tabular}

Note. - The estimates $\left(\hat{\varphi}_{A \rightarrow G}\right.$ and $\left.\hat{\varphi}_{R \rightarrow Q}\right)$ are posterior means in the BPP analysis of genomic data from all six species (Flouris et al., 2019, table 1), while the lower limits for $\varphi_{A \rightarrow G}$ and $\varphi_{R \rightarrow Q}$ are calculated using triplet data (AQG and RQA) using equations for UPGMA and majority-vote $(\mathrm{M}-\mathrm{V})$ respectively.

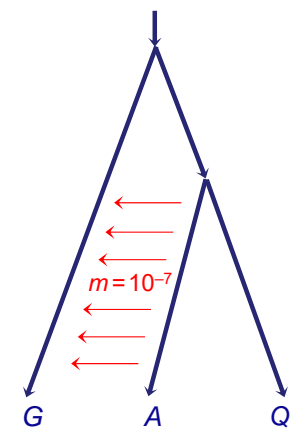

(a) True species history

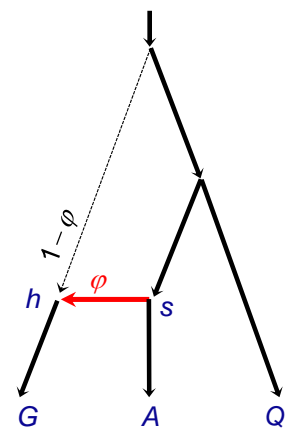

(b) Major species tree

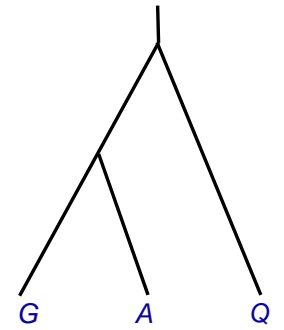

(c) Dominant gene tree

Fig. 9. (a) A plausible species history for A. arabiensis (A), A. quadriannulatus (Q) and A. gambiae (G), with continuous gene flow at the migration rate of $m_{A \rightarrow G} \approx 10^{-7}$. (b) The inferred history when the introgression model is fitted to the genomic (autosomal) sequence data, with the introgression probability $\varphi_{A \rightarrow G}>90 \%$ (table 1). The major species tree is represented by the thick branches. (b) The dominant gene tree. Parameter values $m_{A \rightarrow G}$ and $\varphi_{A \rightarrow G}$ are based on rough calculations using the estimates from the genomic sequence data (see text for discussions).

of species divergence history. As mentioned in Introduction, we have assumed in this paper the backbone species tree to be the true species tree, whether the estimated introgression probability is below or above $50 \%$. If the model of introgression/hybridization applies, it is reasonable to use the introgression probability at each hybridization node to define the "major hybridization branch" and the "major species tree", as in Solis-Lemus et al. (2017). The interpretations of our results will then have to be adjusted accordingly. For example, Figure 2a shows that at 
$\tau_{T} / \tau_{R}=0.4$ under the outflow model, $\mathbb{P}\left(G_{1}\right)<\mathbb{P}\left(G_{2}\right)$ if and only if $\varphi>0.536$. With the true species tree defined to be the major species tree, majority-vote will be consistent when $\varphi>0.536$ (as well as when $\varphi<0.5$ ); it is inconsistent only when $0.5<\varphi<0.536$. Note that the results in Figures 2, 3, 4 and 5 concerning the $\varphi$ and $M$ limits are all valid, but the change of the definition of the true species tree may change the consistency or inconsistency of the methods.

Our analysis in this paper has been motivated by the inferred patterns of gene flow and species divergences in the Anopheles gambiae species complex, where the introgression or hybridization model appears to be a poor fit, and the major species tree for the autosomes does not appear to reflect the true history of species divergence and gene flow (Fontaine et al., 2015; Thawornwattana et al., 2018). Gene flow from A. arabiensis to A. gambiae (or A. coluzzii) affecting the autosomes appears to be ongoing (Slotman et al., 2005), and the continuous migration model may be a more realistic description than the episodic introgression model. Estimates of the migration rate $M_{A \rightarrow G}$ from the $3 \mathrm{~S}$ program vary among the chromosomal arms with the estimate from the combined autosomal data to be 0.22 migrants per generation. In contrast the estimated rate in the opposite direction is $M_{G \rightarrow A}=0$ for all chromosomal arms (Thawornwattana et al., 2018, table S3), consistent with the experiment of Slotman et al. (2005), which was unsuccessful in introducing $A$. gambiae into the A. arabiensis host. Here we use the estimate $M_{A \rightarrow G}=0.22$ as well as the scaled population size $\theta_{G}=4 N_{G} \mu=0.027$ to calculate the migration rate or the proportion of migrants per generation, $m_{A \rightarrow G}$. Suppoose the mutation rate is $2 \times 10^{-9}$ per site per generation based on fruit flies (Keightley et al., 2014). Then we have $N_{G}=0.027 /\left(4 \times 2 \times 10^{-9}\right)=3.4 \times 10^{6}$, and $m_{A \rightarrow G}=M_{A \rightarrow G} / N_{G}=6.5 \times 10^{-8}$ per generation. We note that the migration rates $m$ and $M$ are averages over evolutionary time scales and reflect the action of natural selection removing introgressed alleles or chromosomes (Martin and Jiggins, 2017): for example, $m$ is very likely to be much lower than the proportion of hybrids in the population. Low levels of gene flow are consistent with the distinct species status, given A. gambiae and A. arabiensis have extensively overlapping geographical distributions, as is the fact that hybridization experiments failed to produce introgression in the reverse A. gambiae $\rightarrow$ A. arabiensis direction (Slotman et al., 2005). Nevertheless, as predicted by our theoretical calculations, even such low levels of migration can produce very high introgression probabilities when the data are analyzed under the MSci model. Indeed the estimates of $\varphi_{A \rightarrow G}$ from the same genomic data are greater than $90 \%$ for every autosomal arm except for the $2 \mathrm{La}$ inversion which has a different history (table 2) (Flouris et al., 2019). The evolutionary history in this species complex may be close to the one depicted in Figure 9a (see Mallet et al., 2016, Fig. 3). A very low migration rate, with the proportion of migrants below one in ten million or with far less than one migrant individual per generation, can have a huge impact on the genetic history of the species, such that a sequence sampled today from $G$ will most likely trace its history to the introgressing parental species A. arabiensis. Both the major species tree and the dominant gene tree (Figure 9b\&c) reflect cross-species migration, even though the species phylogeny describing the order of species divergences is undoubtedly the one in Figure 9a.

One may imagine a situation in which the whole genome, including sex chromosomes, are affected by continuous migration, and the whole genome may consistently support the incorrect species phylogeny (Mallet et al., 2016, Fig. 3). It appears that even in such cases, the true history of species divergences including introgression or migration events may still be recoverable using genomic sequence data, given that the IM and MSci models (such as the models in Fig. 9a\&b) make very different predictions on the distribution of gene trees (topologies and coalescent times).

\section{ACKNOWLEDGEMENTS}

We thank three anonymous reviewers, the Associate Editor and Editor for a number of constructive comments. This study has been supported by Biotechnological and Biological Sciences Research Council grants (BB/N000609/1, BB/P006493/1) to Z.Y. and a BBSRC equipment grant (BB/R01356X/1). 


\section{REFERENCES}

Allman, E. S., Bannos, H., and Rhodes, J. A. 2019. Nanuq: A method for inferring species networks from gene trees under the coalescent model. arXive.

Anderson, E. 1949. Introgressive Hydridization. John Wiley, New York.

Chan, Y. C., Roos, C., Inoue-Murayama, M., Inoue, E., Shih, C. C., Pei, K. J., and Vigilant, L. 2013. Inferring the evolutionary histories of divergences in Hylobates and Nomascus gibbons through multilocus sequence data. BMC Evol. Biol., 13: 82.

Dalquen, D., Zhu, T., and Yang, Z. 2017. Maximum likelihood implementation of an isolation-with-migration model for three species. Syst. Biol., 66: 379-398.

Degnan, J. H. 2018. Modeling hybridization under the network multispecies coalescent. Syst. Biol., 67(5): 786-799.

Durand, E. Y., Patterson, N., Reich, D., and Slatkin, M. 2011. Testing for ancient admixture between closely related populations. Mol. Bio.l Evol., 28: 2239-2252.

Edelman, N. B., Frandsen, P. B., Miyagi, M., Clavijo, B., Davey, J., Dikow, R. B., Garcia-Accinelli, G., Van Belleghem, S. M., Patterson, N., Neafsey, D. E., Challis, R., Kumar, S., Moreira, G. R. P., Salazar, C., Chouteau, M., Counterman, B. A., Papa, R., Blaxter, M., Reed, R. D., Dasmahapatra, K. K., Kronforst, M., Joron, M., Jiggins, C. D., McMillan, W. O., Di Palma, F., Blumberg, A. J., Wakeley, J., Jaffe, D., and Mallet, J. 2019. Genomic architecture and introgression shape a butterfly radiation. Science, 366(6465): 594-599.

Ellegren, H., Smeds, L., Burri, R., Olason, P. I., Backstrom, N., Kawakami, T., Kunstner, A., Makinen, H., Nadachowska-Brzyska, K., Qvarnstrom, A., Uebbing, S., and Wolf, J. B. W. 2012. The genomic landscape of species divergence in Ficedula flycatchers. Nature, 491: 756-760.

Flouris, T., Jiao, X., Rannala, B., and Yang, Z. 2018. Species tree inference with bpp using genomic sequences and the multispecies coalescent. Mol. Biol. Evol., 35(10): 2585-2593.

Flouris, T., Jiao, X., Rannala, B., and Yang, Z. 2019. A bayesian implementation of the multispecies coalescent model with introgression for comparative genomic analysis. $J$., page in press.

Folk, R. A., Soltis, P. S., Soltis, D. E., and Guralnick, R. 2018. New prospects in the detection and comparative analysis of hybridization in the tree of life. Am. J. Bot., 105(3): 364-375.

Fontaine, M. C., Pease, J. B., Steele, A., Waterhouse, R. M., Neafsey, D. E., Sharakhov, I. V., Jiang, X., Hall, A. B., Catteruccia, F., Kakani, E., Mitchell, S. N., Wu, Y. C., Smith, H. A., Love, R. R., Lawniczak, M. K., Slotman, M. A., Emrich, S. J., Hahn, M. W., and Besansky, N. J. 2015. Extensive introgression in a malaria vector species complex revealed by phylogenomics. Science, 347(6217): 1258524.

Green, R. E., Krause, J., Briggs, A. W., Maricic, T., Stenzel, U., Kircher, M., Patterson, N., Li, H., Zhai, W., Fritz, M. H., Hansen, N. F., Durand, E. Y., Malaspinas, A. S., Jensen, J. D., Marques-Bonet, T., Alkan, C., Prufer, K., Meyer, M., Burbano, H. A., Good, J. M., Schultz, R., Aximu-Petri, A., Butthof, A., Hober, B., Hoffner, B., Siegemund, M., Weihmann, A., Nusbaum, C., Lander, E. S., Russ, C., Novod, N., Affourtit, J., Egholm, M., Verna, C., Rudan, P., Brajkovic, D., Kucan, Z., Gusic, I., Doronichev, V. B., Golovanova, L. V., Lalueza-Fox, C., de la Rasilla, M., Fortea, J., Rosas, A., Schmitz, R. W., Johnson, P. L., Eichler, E. E., Falush, D., Birney, E., Mullikin, J. C., Slatkin, M., Nielsen, R., Kelso, J., Lachmann, M., Reich, D., and Paabo, S. 2010. A draft sequence of the neandertal genome. Science, 328: 710-722. 
Hahn, M. W. and Nakhleh, L. 2016. Irrational exuberance for resolved species trees. Evolution, 70(1): 7-17.

Heled, J. and Drummond, A. J. 2010. Bayesian inference of species trees from multilocus data. Mol. Biol. Evol., 27: 570-580.

Hey, J. 2010. Isolation with migration models for more than two populations. Mol. Biol. Evol., 27: 905-920.

Hey, J. and Nielsen, R. 2004. Multilocus methods for estimating population sizes, migration rates and divergence time, with applications to the divergence of Drosophila pseudoobscura and D. persimilis. Genetics, 167: 747-760.

Hey, J., Chung, Y., Sethuraman, A., Lachance, J., Tishkoff, S., Sousa, V. C., and Wang, Y. 2018. Phylogeny estimation by integration over isolation with migration models. Mol. Biol. Evol., 35(11): 2805-2818.

Hobolth, A., Andersen, L., and Mailund, T. 2011. On computing the coalescence time density in an isolation-with-migration model with few samples. Genetics, 187: 1241-1243.

Hudson, R. 1983. Testing the constant-rate neutral alele model with protein sequence data. Evolution, 37: 203-217.

Jukes, T. and Cantor, C. 1969. Evolution of protein molecules. In H. Munro, editor, Mammalian Protein Metabolism, pages 21-123. Academic Press, New York.

Keightley, P. D., Ness, R. W., Halligan, D. L., and Haddrill, P. R. 2014. Estimation of the spontaneous mutation rate per nucleotide site in a drosophila melanogaster full-sib family. Genetics, 196(1): 313-320.

Kumar, V., Lammers, F., Bidon, T., Pfenninger, M., Kolter, L., Nilsson, M. A., and Janke, A. 2017. The evolutionary history of bears is characterized by gene flow across species. Sci Rep, 7: 46487.

Leaché, A. D., Harris, R. B., Rannala, B., and Yang, Z. 2014. The influence of gene flow on bayesian species tree estimation: A simulation study. Syst. Biol., 63(1): 17-30.

Liu, L. and Pearl, D. K. 2007. Species trees from gene trees: reconstructing bayesian posterior distributions of a species phylogeny using estimated gene tree distributions. Syst. Biol., 56(3): 504-514.

Liu, L. and Yu, L. 2011. Estimating species trees from unrooted gene trees. Syst. Biol., 60: 661-667.

Liu, L., Yu, L., Pearl, D. K., and Edwards, S. V. 2009. Estimating species phylogenies using coalescence times among sequences. Syst. Biol., 58: 468-477.

Long, C. and Kubatko, L. 2018. The effect of gene flow on coalescent-based species-tree inference. Syst. Biol., 67(5): 770-785.

Maddison, W. 1997. Gene trees in species trees. Syst. Biol., 46: 523-536.

Mallet, J. 2007. Hybrid speciation. Nature, 446: 279-283.

Mallet, J., Besansky, N., and Hahn, M. W. 2016. How reticulated are species? BioEssays, 38(2): $140-149$.

Mao, Y., Economo, E. P., and Satoh, N. 2018. The roles of introgression and climate change in the rise to dominance of Acropora corals. Curr. Biol., 28(21): 3373-3382 e5. 
Martin, S. H. and Jiggins, C. D. 2017. Interpreting the genomic landscape of introgression. Curr. Opin. Genet. Dev., 47: 69-74.

Mirarab, S., Reaz, R., Bayzid, M. S., Zimmermann, T., Swenson, M. S., and Warnow, T. 2014. Astral: genome-scale coalescent-based species tree estimation. Bioinformatics, 30(17): i541-548.

Nichols, R. 2001. Gene trees and species trees are not the same. Trends Ecol. Evol., 16: 358-364.

Nielsen, R., Akey, J. M., Jakobsson, M., Pritchard, J. K., Tishkoff, S., and Willerslev, E. 2017. Tracing the peopling of the world through genomics. Nature, 541: 302.

Slotman, M. A., Della Torre, A., Calzetta, M., and Powell, J. R. 2005. Differential introgression of chromosomal regions between Anopheles gambiae and An. arabiensis. Am. J. Trop. Med. Hyg., 73(2): 326-335.

Solis-Lemus, C. and Ane, C. 2016. Inferring phylogenetic networks with maximum pseudolikelihood under incomplete lineage sorting. PLoS Genet., 12(3): e1005896.

Solis-Lemus, C., Yang, M., and Ane, C. 2016. Inconsistency of species tree methods under gene flow. Syst. Biol., 65(5): 843-851.

Solis-Lemus, C., Bastide, P., and Ane, C. 2017. Phylonetworks: A package for phylogenetic networks. Mol. Biol. Evol., 34(12): 3292-3298.

Thawornwattana, Y., Dalquen, D., and Yang, Z. 2018. Coalescent analysis of phylogenomic data confidently resolves the species relationships in the Anopheles gambiae species complex. Mol. Biol. Evol., 35(10): 2512-2527.

Wen, D. and Nakhleh, L. 2018. Coestimating reticulate phylogenies and gene trees from multilocus sequence data. Syst. Biol., 67(3): 439-457.

Wen, D., Yu, Y., and Nakhleh, L. 2016. Bayesian inference of reticulate phylogenies under the multispecies network coalescent. PLoS Genet., 12(5): e1006006.

Wiebe, A., Longbottom, J., Gleave, K., Shearer, F. M., Sinka, M. E., Massey, N. C., Cameron, E., Bhatt, S., Gething, P. W., Hemingway, J., Smith, D. L., Coleman, M., and Moyes, C. L. 2017. Geographical distributions of African malaria vector sibling species and evidence for insecticide resistance. Malar. J., 16(1): 85.

Wilkinson-Herbots, H. M. 2012. The distribution of the coalescence time and the number of pairwise nucleotide differences in a model of population divergence or speciation with an initial period of gene flow. Theor. Popul. Biol., 82: 92-108.

Wu, D.-D., Ding, X.-D., Wang, S., Wojcik, J. M., Zhang, Y., Tokarska, M., Li, Y., Wang, M.-S., Faruque, O., Nielsen, R., Zhang, Q., and Zhang, Y.-P. 2018. Pervasive introgression facilitated domestication and adaptation in the bos species complex. Nature Ecol. Evol., 2(7): 1139-1145.

$\mathrm{Xu}, \mathrm{B}$. and Yang, Z. 2016. Challenges in species tree estimation under the multispecies coalescent model. Genetics, 204: 1353-1368. doi: 10.1534/genetics.116.190173.

Yang, Z. 1994. Statistical properties of the maximum likelihood method of phylogenetic estimation and comparison with distance matrix methods. Syst. Biol., 43: 329-342.

Yang, Z. 2000. Complexity of the simplest phylogenetic estimation problem. Proc. R. Soc. B: Biol. Sci., 267: 109-116. 
Yang, Z. 2002. Likelihood and Bayes estimation of ancestral population sizes in hominoids using data from multiple loci. Genetics, 162(4): 1811-1823.

Yang, Z. 2007. Paml 4: Phylogenetic analysis by maximum likelihood. Mol. Biol. Evol., 24: 1586-1591.

Yang, Z. 2015. The BPP program for species tree estimation and species delimitation. Curr. Zool., 61: 854-865.

Yang, Z. and Rannala, B. 2014. Unguided species delimitation using DNA sequence data from multiple loci. Mol. Biol. Evol., 31(12): 3125-3135.

Yu, Y. and Nakhleh, L. 2015. A maximum pseudo-likelihood approach for phylogenetic networks. BMC Genomics, 16 Suppl 10: S10.

Yu, Y., Degnan, J. H., and Nakhleh, L. 2012. The probability of a gene tree topology within a phylogenetic network with applications to hybridization detection. PLoS Genet., 8(4): e1002660.

Yu, Y., Dong, J., Liu, K. J., and Nakhleh, L. 2014. Maximum likelihood inference of reticulate evolutionary histories. Proc. Natl. Acad. Sci. U.S.A., 111(46): 16448-16453.

Zhang, C., Ogilvie, H. A., Drummond, A. J., and Stadler, T. 2018. Bayesian inference of species networks from multilocus sequence data. Mol. Biol. Evol., 35: 504-517.

Zhu, J., Yu, Y., and Nakhleh, L. 2016. In the light of deep coalescence: revisiting trees within networks. BMC Bioinformatics, 17: 415.

Zhu, S. and Degnan, J. H. 2017. Displayed trees do not determine distinguishability under the network multispecies coalescent. Syst. Biol., 66(2): 283-298.

Zhu, T. and Yang, Z. 2012. Maximum likelihood implementation of an isolation-with-migration model with three species for testing speciation with gene flow. Mol. Biol. Evol., 29: 3131-3142. 